\title{
Bifurcation Study on Fractional-Order Cohen-Grossberg Neural Networks Involving Delays
}

\author{
Bingnan Tang (D) \\ Business School, Jiangsu University of Technology, Changzhou 213001, China \\ Correspondence should be addressed to Bingnan Tang; regales1988@sina.com
}

Received 28 August 2020; Revised 18 September 2020; Accepted 24 September 2020; Published 15 October 2020

Academic Editor: Chuangxia Huang

Copyright (c) 2020 Bingnan Tang. This is an open access article distributed under the Creative Commons Attribution License, which permits unrestricted use, distribution, and reproduction in any medium, provided the original work is properly cited.

This work is chiefly concerned with the stability behavior and the appearance of Hopf bifurcation of fractional-order delayed Cohen-Grossberg neural networks. Firstly, we study the stability and the appearance of Hopf bifurcation of the involved neural networks with identical delay $\vartheta_{1}=\vartheta_{2}=\vartheta$. Secondly, the sufficient criterion to guarantee the stability and the emergence of Hopf bifurcation for given neural networks with the delay $\vartheta_{2}=0$ is set up. Thirdly, we derive the sufficient condition ensuring the stability and the appearance of Hopf bifurcation for given neural networks with the delay $\vartheta_{1}=0$. The investigation manifests that the delay plays a momentous role in stabilizing networks and controlling the Hopf bifurcation of the addressed fractional-order delayed neural networks. At last, software simulation results successfully verified the rationality of the analytical results. The theoretical findings of this work can be applied to design, control, and optimize neural networks.

\section{Introduction}

It is common knowledge that neural networks have broad application perspectives in a number of subjects such as data science, biology, artificial intelligence, control engineering, biomedical treatment, and so on [1-6]. During a long period, in order to further grasp the inherent laws and dynamic characteristics of neural networks, a great deal of scholars have made great efforts to study neural networks and fruitful results have constantly been emerging. For instance, Hajihosseini et al. [7] investigated the Hopf bifurcation for a class of delayed neural networks by applying the frequency domain approach. In [8], Lin et al. [8] dealt with Turing instability and Turing-Hopf bifurcation for a kind of reactiondiffusion neural networks with leakage term. In 2019, Aouiti et al. [9] established the sufficient condition to ensure the existence and exponential stability of piecewise pseudo almost periodic solution for delayed inertial neural networks with impulse. In 2020, Xu et al. [10] obtained new results on pseudo almost periodic solutions for quaternion-valued delayed fuzzy cellular neural networks. Kumar and Das [11] made a detailed discussion on exponential stability of delayed BAM neural network by applying the matrix measure method. For more related knowledge on these dynamics of neural networks, we refer the readers to [12-23].

It is worth pointing out that all the mentioned works are concerned with the integer-order neural networks. For a long time in the past, the development of fractional calculus has been kept in a very low state due to the lack of practical background and the imperfection of the theoretical system. In recent decades, fractional calculus has been found to have wide applications in numerous fields such as electromagnetic waves, biological engineering, control theory, network science, and so on [24-27]. The fractional calculus has greater advantages than integer-order ones since it can better portray memory nature and hereditary information for the dynamical systems in natural world [24-27]. Nowadays, many scholars pay much attention to the fractional-order dynamical models. Especially, the study on fractional-order neural networks has aroused the close attention of many scholars. Many outstanding achievements on fractionalorder neural networks are available (see [28-31]).

Hopf bifurcation, which acts as a vital dynamical phenomenon of neural networks, naturally arouses the extensive research interest from plenty of scholars. During the past 
several decades, lots of researchers only deal with the Hopf bifurcation for integer-order neural networks. However, the investigation on Hopf bifurcation for fractional-order ones is very scarce. At present, some works on Hopf bifurcation for fractional-order neural networks have been reported. For example, Eshaghi et al. [32] discussed the Hopf bifurcation, chaos control, and synchronization for a chaotic fractionalorder model. Yuan et al. [33] obtained the new results on bifurcation for fractional-order complex-valued delayed neural networks. Xiao et al. [34] dealt with the fractionalorder PD control of Hopf bifurcations for delayed fractionalorder small-world networks. For more detailed publications, one can see [35-38]. Our concern arises: what influence do different delays have on the stability peculiarity and bifurcation phenomenon of the involved neural networks? The answer will play a key role in controlling neural networks. Inspired by this viewpoint, in this present work, we shall deal with the Hopf bifurcation of delayed neural networks by selecting different delays as bifurcation parameters. Detailed works include the following points: (a) probe into the influence of delay on stability and Hopf bifurcation of fractional-order Cohen-Grossberg neural networks with delays and (b) establish some new sufficient conditions to ensure the stability and the appearance of Hopf bifurcation for fractional-order Cohen-Grossberg neural networks by regarding different delays as bifurcation parameters.

In [39], Liu and Xu studied the following integer-order delayed Cohen-Grossberg neural networks:

$$
\left\{\begin{array}{l}
\frac{\mathrm{d} u_{1}(t)}{\mathrm{d} t}=-\alpha_{1}\left(u_{1}(t)\right)\left[\beta_{1}\left(u_{1}(t)\right)-\gamma_{1} h_{2}\left(u_{2}\left(t-\vartheta_{2}\right)\right)\right], \\
\frac{\mathrm{d} u_{2}(t)}{\mathrm{d} t}=-\alpha_{2}\left(u_{2}(t)\right)\left[\beta_{2}\left(u_{2}(t)\right)-\gamma_{2} h_{1}\left(u_{1}\left(t-\vartheta_{1}\right)\right)\right],
\end{array}\right.
$$

where $u_{j}(t)$ stands for the state of the $j$ th neuron; $\alpha_{j}(\cdot)$ stands for the amplification function; $\beta_{j}(\cdot)$ denotes the behaved function; $\gamma_{j}$ denotes the connection weight; $h_{j}(\cdot)$ represents the signal function of the $j$ th neuron at time $t$; and $\vartheta_{j}$ is a delay that corresponds to the finite speed of the axonal signal transmission at time $t, j=1,2$ [39]. By selecting the sum of two delays as bifurcation parameter, the authors established the sufficient conditions to guarantee the stability and the emergence of Hopf bifurcation of model (1). In addition, with the aid of the center manifold theory and the normal form knowledge, the direction and stability of bifurcating periodic solutions are analyzed. Inspired by the analysis above, on the basis of model (1), we establish the following fractional-order version of model (1):

$$
\left\{\begin{array}{l}
\frac{\mathrm{d}^{\psi} u_{1}(t)}{\mathrm{d} t^{\psi}}=-\alpha_{1}\left(u_{1}(t)\right)\left[\beta_{1}\left(u_{1}(t)\right)-\gamma_{1} h_{2}\left(u_{2}\left(t-\vartheta_{2}\right)\right)\right] \\
\frac{\mathrm{d}^{\psi} u_{2}(t)}{\mathrm{d} t^{\psi}}=-\alpha_{2}\left(u_{2}(t)\right)\left[\beta_{2}\left(u_{2}(t)\right)-\gamma_{2} h_{1}\left(u_{1}\left(t-\vartheta_{1}\right)\right)\right]
\end{array}\right.
$$

where $u_{j}(t)$ stands for the state of the $j$ th neuron; $\alpha_{j}(\cdot)$ stands for the amplification function; $\beta_{j}(\cdot)$ denotes the behaved function; $\gamma_{j}$ denotes the connection weight; $h_{j}(\cdot)$ represents the signal function of the $j$ th neuron at time $t ; \vartheta_{j}$ is a delay that corresponds to the finite speed of the axonal signal transmission at time $t, j=1,2[39]$; and $\psi \in(0,1)$ is a constant.

In order to find the key results of this work, we first give the assumptions as follows:

(i) $(Q 1) \beta_{j}(0)=0, h_{j}(0)=0, j=1,2$.

(ii) ( $Q 2) \exists \underline{\alpha}_{j}$ and $\bar{\alpha}_{j}$ such that $0<\underline{\alpha}_{j} \leq \alpha_{j} \leq \bar{\alpha}_{j}, j=1,2$.

This paper is structured as follows. Section 2 gives some related knowledge on fractional differential systems. In Sections 3-5, the stability behavior and the emergence of Hopf bifurcation for neural networks (2) with three different cases are investigated. In Section 6, three examples are prepared to indicate the rationality of the established key conclusions and bifurcation diagrams are clearly displayed. Section 7 concludes the paper.

\section{Basic Theory}

In this part, some necessary knowledge on fractional calculus is prepared.

Definition 1 (see [40]). The Caputo fractional-order derivative can be defined in the following:

$$
\mathscr{D}^{\psi} h(\varrho)=\frac{1}{\Gamma(n-\psi)} \int_{\varrho_{0}}^{\varrho} \frac{h^{(l)}(\nu)}{(\varrho-\nu)^{\psi-l+1}} \mathrm{~d} \nu,
$$

where $h(\varrho) \in\left(\varrho_{0}, \infty\right), R, \Gamma(\nu)=\int_{0}^{\infty} \varrho^{\nu-1} e^{-\varrho} \mathrm{d} \varrho, \varrho \geq \varrho_{0}$, and $l \in Z^{+}, l-1 \leq \psi<l$.

The Laplace transform of $\mathscr{D}^{\psi}$ is given by

$$
\begin{gathered}
\mathscr{L}\left\{\mathscr{D}^{\psi} h(t) ; s\right\}=s^{\psi} \mathscr{H}(s)-\sum_{k=0}^{i-1} s^{\psi-k-1} w^{(k)}(0), \\
i-1 \leq \psi<i \in Z^{+},
\end{gathered}
$$

where $\mathscr{H}(s)=\mathscr{L}\{h(t)\}$. If $h^{(k)}(0)=0, k=1,2, \ldots, i$, then $\mathscr{L}\left\{\mathscr{D}^{\psi} h(t) ; s\right\}=s^{\psi} \mathscr{H}(s)$.

Definition 2 (see [41]). We say that $\left(u_{10}, u_{20}\right)$ is an equilibrium point of system (2) provided that

$$
\left\{\begin{array}{l}
-\alpha_{1}\left(u_{10}\right)\left[\beta_{1}\left(u_{10}\right)-\gamma_{1} h_{2}\left(u_{20}\right)\right]=0 \\
-\alpha_{2}\left(u_{20}\right)\left[\beta_{2}\left(u_{20}\right)-\gamma_{2} h_{1}\left(u_{10}\right)\right]=0
\end{array}\right.
$$

Lemma 1 (see $[42,43])$. Let $u_{*}$ be the equilibrium point of the following system:

$$
\frac{\mathrm{d}^{\psi} u(t)}{\mathrm{d} t^{\psi}}=h(t, u(t)), u(0)=u_{0},
$$

where $\psi \in(0,1)$ and $h(t, u(t)): R^{+} \times R^{m} \longrightarrow R^{m} . u_{*}$ is locally asymptotically stable provided that total eigenvalues $\vartheta$ of $\left.(\partial h(t, u) / \partial u)\right|_{u=u_{*}}$ satisfy $|\arg (\vartheta)|>(\psi \pi / 2)$. 
Lemma 2 (see [44]). Consider the system

$$
\left\{\begin{array}{l}
\frac{\mathrm{d}^{\psi_{1}} \mathscr{W}_{1}(t)}{\mathrm{d} t^{\psi_{1}}}=k_{11} \mathscr{W}_{1}\left(t-\vartheta_{11}\right)+k_{12} \mathscr{W}_{2}\left(t-\vartheta_{12}\right)+\cdots+k_{1 n} \mathscr{W}_{m}\left(t-\vartheta_{1 n}\right), \\
\frac{\mathrm{d}^{\psi_{2}} \mathscr{W}_{2}(t)}{\mathrm{d} t^{\psi_{2}}}=k_{21} \mathscr{W}_{1}\left(t-\vartheta_{21}\right)+k_{22} \mathscr{W}_{2}\left(t-\vartheta_{22}\right)+\cdots+k_{2 n} \mathscr{W}_{m}\left(t-\vartheta_{2 n}\right), \\
\vdots \\
\frac{\mathrm{d}^{\psi_{n}} \mathscr{W}_{n}(t)}{\mathrm{d} t^{\psi_{n}}}=k_{n 1} \mathscr{W}_{1}\left(t-\vartheta_{n 1}\right)+k_{n 2} \mathscr{W}_{2}\left(t-\vartheta_{n 2}\right)+\cdots+k_{n n} \mathscr{W}_{n}\left(t-\vartheta_{n n}\right),
\end{array}\right.
$$

where $0<\psi_{j}<1(j=1,2, \ldots, n)$, the initial conditions $\mathscr{W}_{j}(t)=\phi_{j}(t) \in C\left[-\max _{j, h} \vartheta_{j h}, 0\right]$, and $t \in\left[-\max _{j, h} \vartheta_{j h}, 0\right]$, $j, h=1,2, \ldots, n$. Set

$$
\Delta(\sigma)=\left[\begin{array}{cccc}
\sigma^{\psi_{1}}-k_{11} e^{-\sigma \vartheta_{11}} & -k_{12} e^{-\sigma \vartheta_{12}} & \cdots & -k_{1 n} e^{-\sigma \vartheta_{1 n}} \\
-k_{21} e^{-\sigma \vartheta_{12}} & \sigma^{\psi_{2}}-k_{22} e^{-\sigma \vartheta_{22}} & \cdots & -k_{2 n} e^{-\sigma \vartheta_{2 n}} \\
\vdots & \vdots & \ddots & \vdots \\
-k_{n 1} e^{-\sigma \vartheta_{n 1}} & -k_{n 2} e^{-\sigma \vartheta_{n 2}} & \cdots & \sigma^{\psi_{n}}-k_{n n} e^{-\sigma \vartheta_{n n}}
\end{array}\right] .
$$

The zero solution of system (8) is Lyapunov asymptotically stable provided that total roots of $\operatorname{det}(\Delta(\sigma))=0$ have negative real parts.

\section{Bifurcation for System \\ (2) with $\boldsymbol{9}_{1}==\mathbf{9}_{2}==\mathbf{9}$}

In this part, we will investigate the stability and the appearance of Hopf bifurcation for system (2) with $\vartheta_{1}=\vartheta_{2}=\vartheta$. In terms of (Q1) and (Q2), it is easy to see that system (2) has a unique equilibrium point $\mathscr{E}(0,0)$. Under the conditions (Q1) and (Q2), system (2) becomes

$$
\left\{\begin{array}{l}
\frac{\mathrm{d}^{\psi} u_{1}(t)}{\mathrm{d} t^{\psi}}=-\alpha_{1}\left(u_{1}(t)\right)\left[\beta_{1}\left(u_{1}(t)\right)-\gamma_{1} h_{2}\left(u_{2}(t-\vartheta)\right)\right], \\
\frac{\mathrm{d}^{\psi} u_{2}(t)}{\mathrm{d} t^{\psi}}=-\alpha_{2}\left(u_{2}(t)\right)\left[\beta_{2}\left(u_{2}(t)\right)-\gamma_{2} h_{1}\left(u_{1}(t-\vartheta)\right)\right] .
\end{array}\right.
$$

The linear system of model (9) around $\mathscr{E}(0,0)$ takes the following form:

$$
\left\{\frac{\mathrm{d}^{\psi} u_{1}(t)}{\mathrm{d} t^{\psi}}=-\alpha_{1}(0) \beta_{1}^{\prime}(0) u_{1}(t)+\alpha_{1}(0) \gamma_{1} h_{2}^{\prime}(0) u_{2}(t-\vartheta), \frac{\mathrm{d}^{\psi} u_{2}(t)}{\mathrm{d} t^{\psi}}=-\alpha_{2}(0) \beta_{2}^{\prime}(0) u_{2}(t)+\alpha_{2}(0) \gamma_{2} h_{1}^{\prime}(0) u_{1}(t-\vartheta) .\right.
$$

Let

$$
\left\{a_{1}=-\alpha_{1}(0) \beta_{1}^{\prime}(0), a_{2}=\alpha_{1}(0) \gamma_{1} h_{2}^{\prime}(0), b_{1}=-\alpha_{2}(0) \beta_{2}^{\prime}(0), b_{2}=\alpha_{2}(0) \gamma_{2} h_{1}^{\prime}(0) .\right.
$$

Then, system (10) becomes

$$
\left\{\begin{array}{l}
\frac{\mathrm{d}^{\psi} u_{1}(t)}{\mathrm{d} t^{\psi}}=a_{1} u_{1}(t)+a_{2} u_{2}(t-\vartheta) \\
\frac{\mathrm{d}^{\psi} u_{2}(t)}{\mathrm{d} t^{\psi}}=b_{1} u_{2}(t)+b_{2} u_{1}(t-\vartheta)
\end{array}\right.
$$

Thus, we obtain the following associated characteristic equation for (12):

$$
\operatorname{det}\left[\begin{array}{cc}
s^{\psi}-a_{1} & -a_{2} e^{-s \vartheta} \\
-b_{2} e^{-s \vartheta} & s^{\psi}-b_{1}
\end{array}\right]=0 .
$$

According to (13), we get

$$
s^{2 \psi}-\left(a_{1}+b_{1}\right) s^{\psi}+a_{1} b_{1}-a_{2} b_{2} e^{-2 s \vartheta}=0 .
$$

Assume that (Q3) $a_{1}+b_{1}<0, a_{1} b_{1}>a_{2} b_{2}$.

Lemma 3. If $\vartheta=0$ and (Q3) is fulfilled, then model (9) is locally asymptotically stable.

Proof. When $\vartheta=0$, then (14) becomes

$$
\lambda^{2}-\left(a_{1}+b_{1}\right) \lambda+a_{1} b_{1}-a_{2} b_{2}=0 .
$$

By (Q3), one knows that all roots $\lambda_{l}$ of (15) satisfy $\left|\arg \left(\lambda_{l}\right)\right|>(\psi \pi / 2)(l=1,2)$. So, Lemma 3 holds. The proof ends.

Suppose that $s=i \xi=\xi(\cos (\pi / 2)+i \sin (\pi / 2))$ is a root of (14). Then, one has

$$
\left\{\begin{array}{l}
a_{2} b_{2} \cos 2 \xi \vartheta=\xi^{2 \psi} \cos \psi \pi-\left(a_{1}+b_{1}\right) \xi^{\psi} \cos \frac{\psi \pi}{2}+a_{1} b_{1} \\
a_{2} b_{2} \sin 2 \xi \vartheta=\xi^{2 \psi} \sin \psi \pi-\left(a_{1}+b_{1}\right) \xi^{\psi} \sin \frac{\psi \pi}{2}
\end{array}\right.
$$

By (16), one has

$$
\begin{aligned}
a_{2}^{2} b_{2}^{2}= & {\left[\xi^{2 \psi} \cos \psi \pi-\left(a_{1}+b_{1}\right) \xi^{\psi} \cos \frac{\psi \pi}{2}+a_{1} b_{1}\right]^{2} } \\
& +\left[\xi^{2 \psi} \sin \psi \pi-\left(a_{1}+b_{1}\right) \xi^{\psi} \sin \frac{\psi \pi}{2}\right]^{2}
\end{aligned}
$$

which can be written in the following form:

$$
\xi^{4 \psi}+c_{3} \xi^{3 \psi}+c_{2} \xi^{2 \psi}+c_{1} \xi^{\psi}+c_{0}=0,
$$

where 


$$
\left\{\begin{array}{l}
c_{0}=a_{1}^{2} b_{1}^{2}-a_{2}^{2} b_{2}^{2}, \\
c_{1}=-2 a_{1} b_{1}\left(a_{1}+b_{1}\right) \cos \frac{\psi \pi}{2}, \\
c_{2}=\left(a_{1}+b_{1}\right)^{2}, \\
c_{3}=-2\left(a_{1}+b_{1}\right)^{2}\left(\cos \psi \pi \cos \frac{\psi \pi}{2}-\sin \psi \pi \sin \frac{\psi \pi}{2}\right) .
\end{array}\right.
$$

Denote

$$
\begin{gathered}
\mathscr{M}_{1}(\xi)=\xi^{4 \psi}+c_{3} \xi^{3 \psi}+c_{2} \xi^{2 \psi}+c_{1} \xi^{\psi}+c_{0}, \\
\mathscr{M}_{2}(\eta)=\eta^{4}+c_{3} \eta^{3}+c_{2} \eta^{2}+c_{1} \eta+c_{0} .
\end{gathered}
$$

In view of Lemma 3.7 of [45], we have the following conclusion.

Lemma 4. (i) If $c_{i}>0(i=0,1,2,3)$ and (Q3) is true, then equation (14) has no root with zero real parts. (ii) If $c_{0}<0$, then equation (14) has at least a pair of purely imaginary roots. (iii) If $c_{0}>0$ and $\exists v_{0}>0$ which satisfies $\mathscr{M}_{1}\left(v_{0}\right)<0$, then equation (14) possesses at least two pairs of purely imaginary roots.

For the proof of this lemma, one can see [45]. Here we omit it.

Assume that equation (14) has four positive real roots $\xi_{h}(h=1,2,3,4)$. By $(16)$, one has

$$
\vartheta_{f}^{l}=\frac{1}{2 \xi_{f}}\left[\arcsin \left(\frac{\xi_{f}^{2 \psi} \sin \psi \pi-\left(a_{1}+b_{1}\right) \xi_{f}^{\psi} \sin (\psi \pi / 2)}{a_{2} b_{2}}\right)+2 l \pi\right],
$$

where $l=0,1,2, \ldots, f=1,2,3,4$. Set

$$
\vartheta_{0}=\min _{f=1,2,3,4}\left\{\vartheta_{f}^{0}\right\}, \xi_{0}=\left.\xi\right|_{\vartheta=\vartheta_{0}} .
$$

Next, the following assumption is prepared.( (24) $\mathscr{A}_{11} \mathscr{A}_{21}+\mathscr{A}_{12} \mathscr{A}_{22}>0$, where

$$
\left\{\begin{array}{l}
\mathscr{A}_{11}=\left(a_{1}+b_{1}\right) \psi \xi_{0}^{\psi-1} \cos \frac{(\psi-1) \pi}{2}-2 \psi \xi_{0}^{2 \psi-1} \cos \frac{(2 \psi-1) \pi}{2}, \\
\mathscr{A}_{12}=\left(a_{1}+b_{1}\right) \psi \xi_{0}^{\psi-1} \sin \frac{(\psi-1) \pi}{2}-2 \psi \xi_{0}^{2 \psi-1} \sin \frac{(2 \psi-1) \pi}{2}, \\
\mathscr{A}_{21}=2 a_{2} b_{2} \xi_{0} \sin 2 \xi_{0} \vartheta_{0}, \\
\mathscr{A}_{22}=2 a_{2} b_{2} \xi_{0} \cos 2 \xi_{0} \vartheta_{0} .
\end{array}\right.
$$

Lemma 5. If $s(\vartheta)=\chi(\vartheta)+i \xi(\vartheta)$ is the root of equation (14) around $\vartheta=\vartheta_{0}$ such that $\chi\left(\vartheta_{0}\right)=0, \xi\left(\vartheta_{0}\right)=\psi_{0}$, then we get $\left.\operatorname{Re}[d s / d \vartheta]\right|_{\vartheta=\vartheta_{0}, \xi=\xi_{0}}>0$.

Proof. In terms of equation (14), one has

$$
2 \psi s^{2 \psi-1} \frac{\mathrm{d} s}{\mathrm{~d} \vartheta}-\left(a_{1}+b_{1}\right) \psi s^{\psi-1} \frac{\mathrm{d} s}{\mathrm{~d} \vartheta}+2 a_{2} b_{2} e^{-2 s \vartheta}\left(\frac{\mathrm{d} s}{\mathrm{~d} \vartheta} \vartheta+s\right)=0 .
$$

Then,

$$
\left[\frac{\mathrm{d} s}{\mathrm{~d} \vartheta}\right]^{-1}=\frac{\left(a_{1}+b_{1}\right) \psi s^{\psi-1}-2 \psi s^{2 \psi-1}}{2 a_{2} b_{2} s e^{-2 s \vartheta}}-\frac{\vartheta}{s}
$$

Thus,

$$
\begin{aligned}
\operatorname{Re}\left[\frac{\mathrm{d} s}{\mathrm{~d} \vartheta}\right]_{\vartheta=\vartheta_{0}, \xi=\xi_{0}}^{-1} & =\operatorname{Re}\left[\frac{\left(a_{1}+b_{1}\right) \psi s^{\psi-1}-2 \psi s^{2 \psi-1}}{2 a_{2} b_{2} s e^{-2 s \vartheta}}\right]_{\vartheta=\vartheta_{0}, \xi=\xi_{0}} \\
& =\frac{\mathscr{A}_{11} \mathscr{A}_{21}+\mathscr{A}_{12} \mathscr{A}_{22}}{\mathscr{A}_{21}^{2}+\mathscr{A}_{22}^{2}}
\end{aligned}
$$

According to (Q4), one gets

$$
\operatorname{Re}\left[\frac{\mathrm{d} s}{\mathrm{~d} \vartheta}\right]_{\vartheta=\vartheta_{0}, \xi=\xi_{0}}^{-1}>0
$$

The proof ends.

On the basis of above analysis, it is easy to establish the following result.

Theorem 1. If (Q1) - (Q4) are true, then the equilibrium point $\mathscr{E}(0,0)$ of system (2) is locally asymptotically stable when $\vartheta \in 0, \vartheta_{0}$ and a Hopf bifurcation will happen around the equilibrium point $\mathscr{E}(0,0)$ if $\vartheta=\vartheta_{0}$.

\section{Bifurcation for System (2) with $\boldsymbol{9}_{2}==0$}

In this part, we will investigate the stability and the appearance of Hopf bifurcation for system (2) with $\vartheta_{2}=0$. In terms of (Q1) and (Q2), it is easy to see that system (2) has a unique equilibrium point $\mathscr{E}(0,0)$. Under the conditions (Q1) and (Q2), system (2) becomes

$$
\left\{\begin{array}{l}
\frac{\mathrm{d}^{\psi} u_{1}(t)}{\mathrm{d} t^{\psi}}=-\alpha_{1}\left(u_{1}(t)\right)\left[\beta_{1}\left(u_{1}(t)\right)-\gamma_{1} h_{2}\left(u_{2}(t)\right)\right] \\
\frac{\mathrm{d}^{\psi} u_{2}(t)}{\mathrm{d} t^{\psi}}=-\alpha_{2}\left(u_{2}(t)\right)\left[\beta_{2}\left(u_{2}(t)\right)-\gamma_{2} h_{1}\left(u_{1}\left(t-\vartheta_{1}\right)\right)\right] .
\end{array}\right.
$$
form:

The linear system of model $(28)$ around $\mathscr{E}(0,0)$ takes the

$$
\left\{\frac{\mathrm{d}^{\psi} u_{1}(t)}{\mathrm{d} t^{\psi}}=-\alpha_{1}(0) \beta_{1}^{\prime}(0) u_{1}(t)+\alpha_{1}(0) \gamma_{1} h_{2}^{\prime}(0) u_{2}(t), \frac{\mathrm{d}^{\psi} u_{2}(t)}{\mathrm{d} t^{\psi}}=-\alpha_{2}(0) \beta_{2}^{\prime}(0) u_{2}(t)+\alpha_{2}(0) \gamma_{2} h_{1}^{\prime}(0) u_{1}\left(t-\vartheta_{1}\right)\right.
$$


That is,

$$
\left\{\begin{array}{l}
\frac{\mathrm{d}^{\psi} u_{1}(t)}{\mathrm{d} t^{\psi}}=a_{1} u_{1}(t)+a_{2} u_{2}(t) \\
\frac{\mathrm{d}^{\psi} u_{2}(t)}{\mathrm{d} t^{\psi}}=b_{1} u_{2}(t)+b_{2} u_{1}\left(t-\vartheta_{1}\right)
\end{array}\right.
$$

where $a_{1}, a_{2}, b_{1}, b_{1}$ are defined by (11). Thus, we obtain the following associated characteristic equation for (30):

$$
\operatorname{det}\left[\begin{array}{cc}
s^{\psi}-a_{1} & -a_{2} \\
-b_{2} e^{-s \vartheta_{1}} & s^{\psi}-b_{1}
\end{array}\right]=0 .
$$

According to (31), we get

$$
s^{2 \psi}-\left(a_{1}+b_{1}\right) s^{\psi}+a_{1} b_{1}-a_{2} b_{2} e^{-s \vartheta_{1}}=0 .
$$

Lemma 6. If $\vartheta_{1}=0$ and (Q3) is fulfilled, then model (28) is locally asymptotically stable.
Proof. When $\vartheta_{1}=0$, then (32) becomes

$$
\lambda^{2}-\left(a_{1}+b_{1}\right) \lambda+a_{1} b_{1}-a_{2} b_{2}=0 .
$$

By (Q3), one knows that all roots $\lambda_{l}$ of (33) satisfy $\left|\arg \left(\lambda_{l}\right)\right|>(\psi \pi / 2)(l=1,2)$. So, Lemma 6 holds. The proof ends.

Suppose that $s=i \varrho=\varrho(\cos (\pi / 2)+i \sin (\pi / 2))$ is a root of (32). Then, one has

$$
\left\{\begin{array}{l}
a_{2} b_{2} \cos \varrho \vartheta_{1}=\varrho^{2 \psi} \cos \psi \pi-\left(a_{1}+b_{1}\right) \varrho^{\psi} \cos \frac{\psi \pi}{2}+a_{1} b_{1}, \\
a_{2} b_{2} \sin \varrho \vartheta_{1}=\varrho^{2 \psi} \sin \psi \pi-\left(a_{1}+b_{1}\right) \varrho^{\psi} \sin \frac{\psi \pi}{2} .
\end{array}\right.
$$

By (34), one has

$$
a_{2}^{2} b_{2}^{2}=\left[\varrho^{2 \psi} \cos \psi \pi-\left(a_{1}+b_{1}\right) \varrho^{\psi} \cos \frac{\psi \pi}{2}+a_{1} b_{1}\right]^{2}+\left[\varrho^{2 \psi} \sin \psi \pi-\left(a_{1}+b_{1}\right) \varrho^{\psi} \sin \frac{\psi \pi}{2}\right]^{2}
$$

which can be written in the following form:

$$
\varrho^{4 \psi}+c_{3} \varrho^{3 \psi}+c_{2} \varrho^{2 \psi}+c_{1} \varrho^{\psi}+c_{0}=0
$$

where $c_{i}(i=0,1,2,3)$ is defined by (19). Denote

$$
\begin{array}{r}
\mathcal{N}_{1}(\varrho)=\varrho^{4 \psi}+c_{3} \varrho^{3 \psi}+c_{2} \varrho^{2 \psi}+c_{1} \varrho^{\psi}+c_{0}, \\
\mathcal{N}_{2}(\zeta)=\zeta^{4}+c_{3} \zeta^{3}+c_{2} \zeta^{2}+c_{1} \zeta+c_{0} .
\end{array}
$$

In view of Lemma 3.7 of [45], we have the following conclusions.

Lemma 7. (i) If $c_{i}>0(i=0,1,2,3)$ and (Q3) is true, then equation (32) has no root with zero real parts. (ii) If $c_{0}<0$, then equation (32) has at least a pair of purely imaginary roots. (iii) If $c_{0}>0$ and $\exists u_{0}>0$ which satisfies $\mathcal{N}_{1}\left(u_{0}\right)<0$, then equation (32) possesses at least two pairs of purely imaginary roots.

For the proof of this lemma, one can see [45]. Here we omit it.

Assume that equation (32) has four positive real roots $\varrho_{1 h}(h=1,2,3,4)$. By $(34)$, one has

$$
\vartheta_{1 f}^{l}=\frac{1}{\varrho_{f}}\left[\arcsin \left(\frac{\varrho_{f}^{2 \psi} \sin \psi \pi-\left(a_{1}+b_{1}\right) \varrho_{f}^{\psi} \sin (\psi \pi / 2)}{a_{2} b_{2}}\right)+2 l \pi\right],
$$

where $l=0,1,2, \ldots, f=1,2,3,4$. Set

$$
\vartheta_{10}=\min _{f=1,2,3,4}\left\{\vartheta_{1 f}^{0}\right\}, \varrho_{0}=\left.\varrho\right|_{\vartheta_{1}=\vartheta_{10}} .
$$

Next, the following assumption is prepared.(Q5) $\mathscr{B}_{11} \mathscr{B}_{21}+\mathscr{B}_{12} \mathscr{B}_{22}>0$, where

$$
\left\{\begin{array}{l}
\mathscr{B}_{11}=\left(a_{1}+b_{1}\right) \psi \varrho_{0}^{\psi-1} \cos \frac{(\psi-1) \pi}{2}-2 \psi \varrho_{0}^{2 \psi-1} \cos \frac{(2 \psi-1) \pi}{2}, \\
\mathscr{B}_{12}=\left(a_{1}+b_{1}\right) \psi \varrho_{0}^{\psi-1} \sin \frac{(\psi-1) \pi}{2}-2 \psi \varrho_{0}^{2 \psi-1} \sin \frac{(2 \psi-1) \pi}{2}, \\
\mathscr{B}_{21}=a_{2} b_{2} \varrho_{0} \sin \varrho_{0} \vartheta_{10}, \\
\mathscr{B}_{22}=a_{2} b_{2} \varrho_{0} \cos \varrho_{0} \vartheta_{10} .
\end{array}\right.
$$

Lemma 8. If $s\left(\vartheta_{1}\right)=\alpha\left(\vartheta_{1}\right)+i \varrho\left(\vartheta_{1}\right)$ is the root of equation (32) around $\vartheta_{1}=\vartheta_{10}$ such that $\alpha\left(\vartheta_{10}\right)=0, \varrho\left(\vartheta_{10}\right)=\varrho_{0}$, then we get $\left.\operatorname{Re}[d s / d \vartheta]\right|_{\vartheta_{1}=\vartheta_{10}, \varrho=\varrho_{0}}>0$.

Proof. In terms of equation (32), one has

$$
2 \psi s^{2 \psi-1} \frac{\mathrm{d} s}{\mathrm{~d} \vartheta_{1}}-\left(a_{1}+b_{1}\right) \psi s^{\psi-1} \frac{\mathrm{d} s}{\mathrm{~d} \vartheta_{1}}+a_{2} b_{2} e^{-s \vartheta_{1}}\left(\frac{\mathrm{d} s}{\mathrm{~d} \vartheta_{1}} \vartheta_{1}+s\right)=0 .
$$

Then,

$$
\left[\frac{\mathrm{d} s}{\mathrm{~d} \vartheta_{1}}\right]^{-1}=\frac{\left(a_{1}+b_{1}\right) \psi s^{\psi-1}-2 \psi s^{2 \psi-1}}{a_{2} b_{2} s e^{-s \vartheta_{1}}}-\frac{\vartheta_{1}}{s} .
$$

Thus, 


$$
\begin{aligned}
\operatorname{Re}\left[\frac{\mathrm{d} s}{\mathrm{~d} \vartheta_{1}}\right]_{\vartheta_{1}=\vartheta_{10}, \varrho=\varrho_{0}}^{-1} & =\operatorname{Re}\left[\frac{\left(a_{1}+b_{1}\right) \psi s^{\psi-1}-2 \psi s^{2 \psi-1}}{a_{2} b_{2} s e^{-s \vartheta_{1}}}\right]_{\vartheta_{1}=\vartheta_{10}, \varrho=\varrho_{0}} \\
& =\frac{\mathscr{B}_{11} \mathscr{B}_{21}+\mathscr{B}_{12} \mathscr{B}_{22}}{\mathscr{B}_{21}^{2}+\mathscr{B}_{22}^{2}} .
\end{aligned}
$$

According to (Q5), one gets

$$
\operatorname{Re}\left[\frac{\mathrm{d} s}{\mathrm{~d} \vartheta_{1}}\right]_{\vartheta_{1}=\vartheta_{10}, \varrho=\varrho_{0}}^{-1}>0 .
$$

The proof ends.

On the basis of above analysis, it is easy to establish the following result.

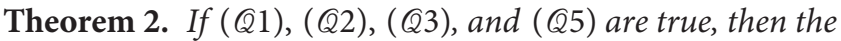
equilibrium point $\mathscr{E}(0,0)$ of system (28) is locally asymptotically stable when $\vartheta_{1} \in 0, \vartheta_{10}$ and a Hopf bifurcation will happen around the equilibrium point $\mathscr{E}(0,0)$ if $\vartheta_{1}=\vartheta_{10}$.

\section{Bifurcation for System (2) with $\boldsymbol{\vartheta}_{1}==0$}

In this part, we will investigate the stability and the appearance of Hopf bifurcation for system (2) with $\vartheta_{1}=0$. In terms of (Q1) and (Q2), it is easy to see that system (2) has a unique equilibrium point $\mathscr{E}(0,0)$. Under the conditions $(Q 1)$ and (Q2), system (2) becomes

$$
\left\{\begin{array}{l}
\frac{\mathrm{d}^{\psi} u_{1}(t)}{\mathrm{d} t^{\psi}}=-\alpha_{1}\left(u_{1}(t)\right)\left[\beta_{1}\left(u_{1}(t)\right)-\gamma_{1} h_{2}\left(u_{2}\left(t-\vartheta_{2}\right)\right)\right], \\
\frac{\mathrm{d}^{\psi} u_{2}(t)}{\mathrm{d} t^{\psi}}=-\alpha_{2}\left(u_{2}(t)\right)\left[\beta_{2}\left(u_{2}(t)\right)-\gamma_{2} h_{1}\left(u_{1}(t)\right)\right] .
\end{array}\right.
$$

The linear system of model $(45)$ around $\mathscr{E}(0,0)$ takes the form

$$
\left\{\frac{\mathrm{d}^{\psi} u_{1}(t)}{\mathrm{d} t^{\psi}}=-\alpha_{1}(0) \beta_{1}^{\prime}(0) u_{1}(t)+\alpha_{1}(0) \gamma_{1} h_{2}^{\prime}(0) u_{2}\left(t-\vartheta_{2}\right), \frac{\mathrm{d}^{\psi} u_{2}(t)}{\mathrm{d} t^{\psi}}=-\alpha_{2}(0) \beta_{2}^{\prime}(0) u_{2}(t)+\alpha_{2}(0) \gamma_{2} h_{1}^{\prime}(0) u_{1}(t) .\right.
$$

That is,

$$
\left\{\begin{array}{l}
\frac{\mathrm{d}^{\psi} u_{1}(t)}{\mathrm{d} t^{\psi}}=a_{1} u_{1}(t)+a_{2} u_{2}\left(t-\vartheta_{2}\right), \\
\frac{\mathrm{d}^{\psi} u_{2}(t)}{\mathrm{d} t^{\psi}}=b_{1} u_{2}(t)+b_{2} u_{1}(t),
\end{array}\right.
$$

where $a_{1}, a_{2}, b_{1}, b_{1}$ are defined by (11). Thus, we obtain the following associated characteristic equation for (47):

$$
\operatorname{det}\left[\begin{array}{cc}
s^{\psi}-a_{1} & -a_{2} e^{-s \vartheta_{2}} \\
-b_{2} & s^{\psi}-b_{1}
\end{array}\right]=0 .
$$

According to (48), we get

$$
s^{2 \psi}-\left(a_{1}+b_{1}\right) s^{\psi}+a_{1} b_{1}-a_{2} b_{2} e^{-s \vartheta_{2}}=0 .
$$

Lemma 9. If $\vartheta_{2}=0$ and (Q3) is fulfilled, then model (45) is locally asymptotically stable.

Proof. When $\vartheta_{2}=0$, then (49) becomes

$$
\lambda^{2}-\left(a_{1}+b_{1}\right) \lambda+a_{1} b_{1}-a_{2} b_{2}=0 .
$$

By (Q3), one knows that all roots $\lambda_{j}$ of (50) satisfy $\left|\arg \left(\lambda_{j}\right)\right|>(\psi \pi / 2)(j=1,2)$. So, Lemma 9 holds. The proof ends.

Suppose that $s=i \sigma=\sigma(\cos (\pi / 2)+i \sin (\pi / 2))$ is a root of (49). Then, one has

$$
\left\{\begin{array}{l}
a_{2} b_{2} \cos \sigma \vartheta_{2}=\sigma^{2 \psi} \cos \psi \pi-\left(a_{1}+b_{1}\right) \sigma^{\psi} \cos (\psi \pi / 2)+a_{1} b_{1}, \\
a_{2} b_{2} \sin \sigma \vartheta_{2}=\sigma^{2 \psi} \sin \psi \pi-\left(a_{1}+b_{1}\right) \sigma^{\psi} \sin (\psi \pi / 2) .
\end{array}\right.
$$

By (51), one has

$$
\begin{aligned}
a_{2}^{2} b_{2}^{2}= & {\left[\sigma^{2 \psi} \cos \psi \pi-\left(a_{1}+b_{1}\right) \sigma^{\psi} \cos (\psi \pi / 2)+a_{1} b_{1}\right]^{2} } \\
& +\left[\sigma^{2 \psi} \sin \psi \pi-\left(a_{1}+b_{1}\right) \sigma^{\psi} \sin (\psi \pi / 2)\right]^{2},
\end{aligned}
$$

which can be written in the following form:

$$
\sigma^{4 \psi}+c_{3} \sigma^{3 \psi}+c_{2} \sigma^{2 \psi}+c_{1} \sigma^{\psi}+c_{0}=0
$$

where $c_{i}(i=0,1,2,3)$ is defined by (19). Denote

$$
\begin{array}{r}
\mathscr{K}_{1}(\sigma)=\sigma^{4 \psi}+c_{3} \sigma^{3 \psi}+c_{2} \sigma^{2 \psi}+c_{1} \sigma^{\psi}+c_{0}, \\
\mathscr{K}_{2}(\varsigma)=\varsigma^{4}+c_{3} \varsigma^{3}+c_{2} \varsigma^{2}+c_{1} \varsigma+c_{0} .
\end{array}
$$

In view of Lemma 3.7 of [45], we have the following conclusions.

Lemma 10. (i) If $c_{i}>0(i=0,1,2,3)$ and (Q3) is true, then equation (49) has no root with zero real parts. (ii) If $c_{0}<0$, then equation (49) has at least a pair of purely imaginary roots. (iii) If $c_{0}>0$ and $\exists w_{0}>0$ which satisfies $\mathscr{K}_{1}\left(w_{0}\right)<0$, then equation (49) possesses at least two pairs of purely imaginary roots.

For the proof of this lemma, one can see [45]. Here we omit it.

Assume that equation (49) has four positive real roots $\varrho_{2 h}(h=1,2,3,4)$. By $(51)$, one has

$$
\vartheta_{2 f}^{l}=\frac{1}{\sigma_{f}}\left[\arcsin \left(\frac{\sigma_{f}^{2 \psi} \sin \psi \pi-\left(a_{1}+b_{1}\right) \sigma_{f}^{\psi} \sin (\psi \pi / 2)}{a_{2} b_{2}}\right)+2 l \pi\right]
$$


where $l=0,1,2, \ldots, f=1,2,3,4$. Set

$$
\vartheta_{20}=\min _{f=1,2,3,4}\left\{\vartheta_{2 f}^{0}\right\}, \sigma_{0}=\left.\sigma\right|_{\vartheta_{2}=\vartheta_{20}} .
$$

Next, the following assumption is prepared.(Q6) $\mathscr{C}_{11} \mathscr{C}_{21}+\mathscr{C}_{12} \mathscr{C}_{22}>0$, where

$$
\left\{\begin{array}{l}
\mathscr{C}_{11}=\left(a_{1}+b_{1}\right) \psi \sigma_{0}^{\psi-1} \cos \frac{(\psi-1) \pi}{2}-2 \psi \sigma_{0}^{2 \psi-1} \cos \frac{(2 \psi-1) \pi}{2}, \\
\mathscr{C}_{12}=\left(a_{1}+b_{1}\right) \psi \sigma_{0}^{\psi-1} \sin \frac{(\psi-1) \pi}{2}-2 \psi \sigma_{0}^{2 \psi-1} \sin \frac{(2 \psi-1) \pi}{2}, \\
\mathscr{C}_{21}=a_{2} b_{2} \sigma_{0} \sin \sigma_{0} \vartheta_{10}, \\
\mathscr{C}_{22}=a_{2} b_{2} \sigma_{0} \cos \sigma_{0} \vartheta_{10} .
\end{array}\right.
$$

Lemma 11. If $s\left(\vartheta_{2}\right)=\beta\left(\vartheta_{2}\right)+i \sigma\left(\vartheta_{2}\right)$ is the root of equation (32) around $\vartheta_{2}=\vartheta_{20}$ such that $\beta\left(\vartheta_{20}\right)=0, \sigma\left(\vartheta_{20}\right)=\varrho_{0}$, then we get $\left.\operatorname{Re}\left[d s / d \vartheta_{2}\right]\right|_{\vartheta_{2}=\vartheta_{20}, \sigma=\sigma_{0}}>0$.

Proof. In terms of equation (49), one has

$$
2 \psi s^{2 \psi-1} \frac{\mathrm{d} s}{\mathrm{~d} \vartheta_{2}}-\left(a_{1}+b_{1}\right) \psi s^{\psi-1} \frac{\mathrm{d} s}{\mathrm{~d} \vartheta_{2}}+a_{2} b_{2} e^{-s \vartheta_{2}}\left(\frac{\mathrm{d} s}{\mathrm{~d} \vartheta_{2}} \vartheta_{2}+s\right)=0 .
$$

Then,

$$
\frac{\mathrm{d} s^{-1}}{\mathrm{~d} \vartheta_{2}}=\frac{\left(a_{1}+b_{1}\right) \psi s^{\psi-1}-2 \psi s^{2 \psi-1}}{a_{2} b_{2} s e^{-s \vartheta_{2}}}-\frac{\vartheta_{2}}{s} .
$$

Thus,

$$
\begin{aligned}
\operatorname{Re}\left[\frac{\mathrm{d} s}{\mathrm{~d} \vartheta_{2}}\right]_{\vartheta_{2}=\vartheta_{20}, \sigma=\sigma_{0}}^{-1}= & \operatorname{Re}\left[\frac{\left(a_{1}+b_{1}\right) \psi s^{\psi-1}-2 \psi s^{2 \psi-1}}{a_{2} b_{2} s e^{-s \vartheta_{2}}}\right]_{\vartheta_{2}=\vartheta_{20}, \sigma=\sigma_{0}} \\
= & \frac{\mathscr{C}_{11} \mathscr{C}_{21}+\mathscr{C}_{12} \mathscr{C}_{22}}{\mathscr{C}_{21}^{2}+\mathscr{C}_{22}^{2}} .
\end{aligned}
$$

According to (Q6), one gets

$$
\operatorname{Re}\left[\frac{\mathrm{d} s}{\mathrm{~d} \vartheta_{2}}\right]_{\vartheta_{2}=\vartheta_{20}, \sigma=\sigma_{0}}^{-1}>0 .
$$

The proof ends.

On the basis of above analysis, it is easy to establish the following result.

Theorem 3. If (Q1), (Q2), (Q3), and (Q6) are true, then the equilibrium point $\mathscr{E}(0,0)$ of system $(45)$ is locally asymptotically stable when $\vartheta_{2} \in 0, \vartheta_{20}$ and a Hopf bifurcation will happen around the equilibrium point $\mathscr{E}(0,0)$ if $\vartheta_{1}=\vartheta_{20}$.
Remark 1. In [39], Liu and Xu have dealt with the bifurcation of integer-order delayed Cohen-Grossberg neural networks (1) by selecting the sum of two delays as bifurcation parameter. They did not consider the impact of two different delays on the stability and Hopf bifurcation of the neural networks (1). In this paper, we set up new fractionalorder delayed Cohen-Grossberg neural networks (2). We have focused on the impact of two different delays on the stability and Hopf bifurcation of the established neural networks (2) by regarding the three different delays ((1) $\vartheta_{1}=\vartheta_{2}=\vartheta$, (2) $\vartheta_{1}$, and (3) $\vartheta_{2}$ ) as bifurcation parameters. Based on this viewpoint, we think that our work is a good complement and improvement of Liu and $\mathrm{Xu}$ [39].

Remark 2. In [2], the authors studied the Hopf bifurcation of integer-order delayed neural networks by the frequency domain approach. Now we cannot investigate the Hopf bifurcation of fractional-order delayed Cohen-Grossberg neural networks by the frequency domain approach.

Remark 3. Now we cannot investigate the direction and stability of Hopf bifurcation of the involved neural networks due to the lack of theoretical tools.

\section{Three Examples}

Example 1. Given the following system:

$\left\{\begin{array}{l}\frac{\mathrm{d}^{\psi} u_{1}(t)}{\mathrm{d} t^{\psi}}=-\left[4.25+\sin u_{1}(t)\right]\left[1.22 u_{1}(t)-1.23 \tanh \left(u_{2}(t-9)\right)\right], \\ \frac{\mathrm{d}^{\psi} u_{2}(t)}{\mathrm{d} t^{\psi}}=-\left[6.13+\sin u_{2}(t)\right]\left[2.13 u_{2}(t)-2.05 \tanh \left(u_{1}(t-9)\right)\right] .\end{array}\right.$

Clearly, system (62) possesses the equilibrium point $\mathscr{E}(0,0)$. Using the computer software, one derives $\varphi_{0}=0.0499$ and $\vartheta_{0}=0.142$ and the hypotheses $(Q 1)-(Q 4)$ in Theorem 1 are fulfilled. So, one knows that $\mathscr{E}(0,0)$ of model (62) maintains locally asymptotically stable state provided that $0 \leq \vartheta<0.142$. Choosing $\vartheta=0.12<\vartheta_{0}=0.142$, we get the simulation plot (Figure 1) which manifests that the states of two neurons will tend to be zero. Model (62) will be unstable, and the Hopf bifurcation phenomenon takes place provided that $\vartheta \geq 0.142$. Selecting $\vartheta=0.23>\vartheta_{0}=0.142$, one gets the simulation plot (Figure 2) which shows that the states of two neurons will keep periodic vibration in the vicinity of $\mathscr{E}(0,0)$. We give bifurcation plots (see Figures 3 and 4 ) to display the bifurcation point. Besides, the quantity change for $\xi_{0}$ and $\vartheta_{0}$ is shown in Table 1 .

Example 2. Given the following system:

$$
\left\{\begin{array}{l}
\frac{\mathrm{d}^{\psi} u_{1}(t)}{\mathrm{d} t^{\psi}}=-\left[4.25+\sin u_{1}(t)\right]\left[1.22 u_{1}(t)-1.23 \tanh \left(u_{2}(t)\right)\right], \\
\frac{\mathrm{d}^{\psi} u_{2}(t)}{\mathrm{d} t^{\psi}}=-\left[6.13+\sin u_{2}(t)\right]\left[2.13 u_{2}(t)-2.05 \tanh \left(u_{1}\left(t-\vartheta_{1}\right)\right)\right] .
\end{array}\right.
$$




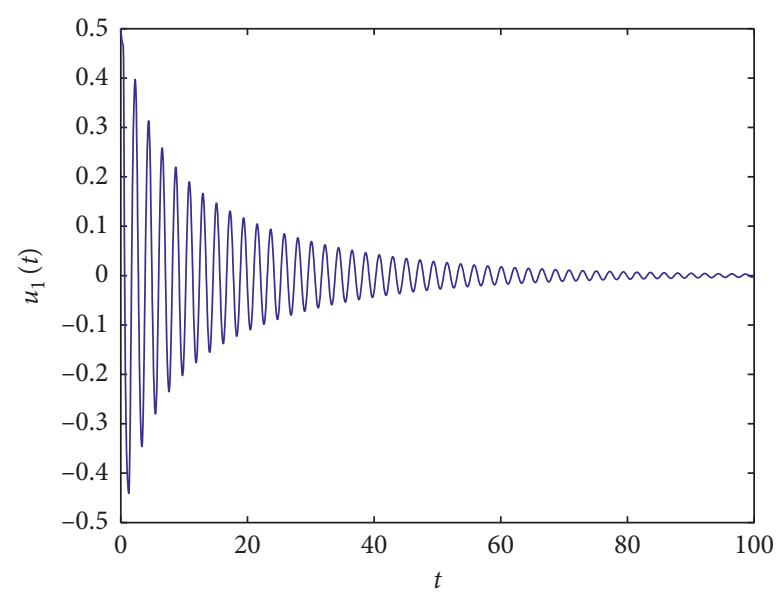

(a)

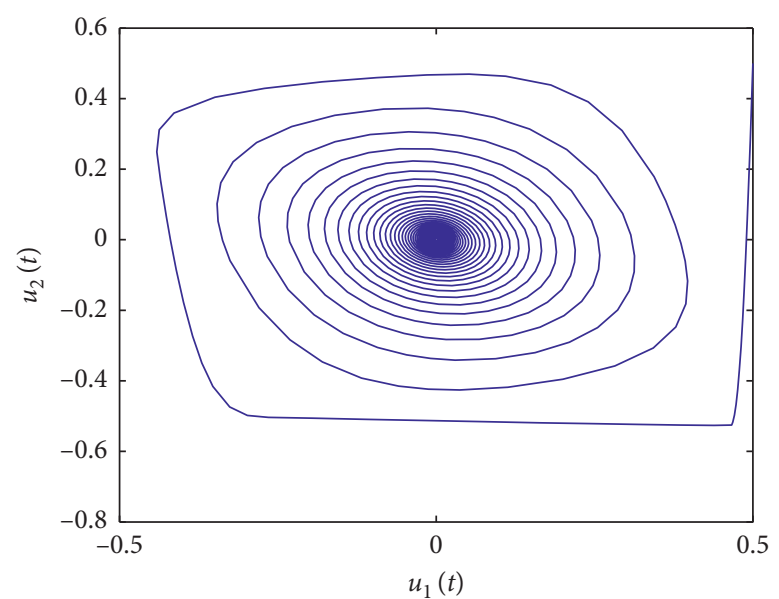

(c)

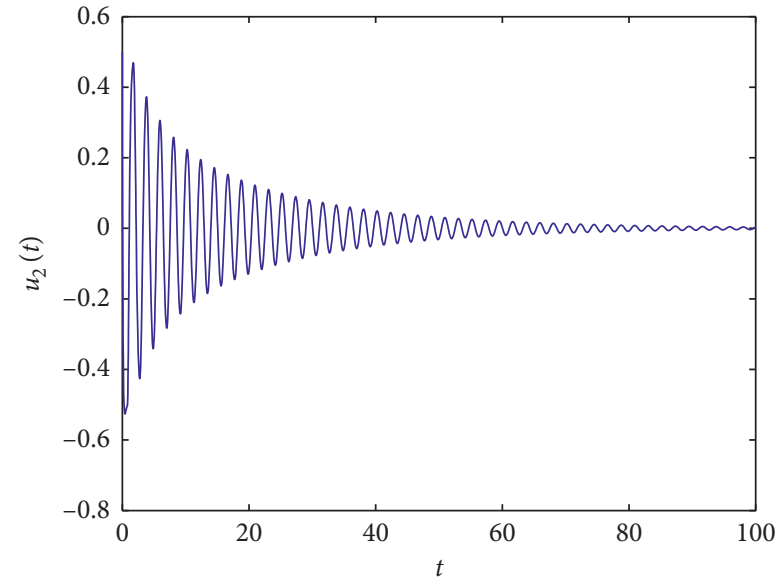

(b)

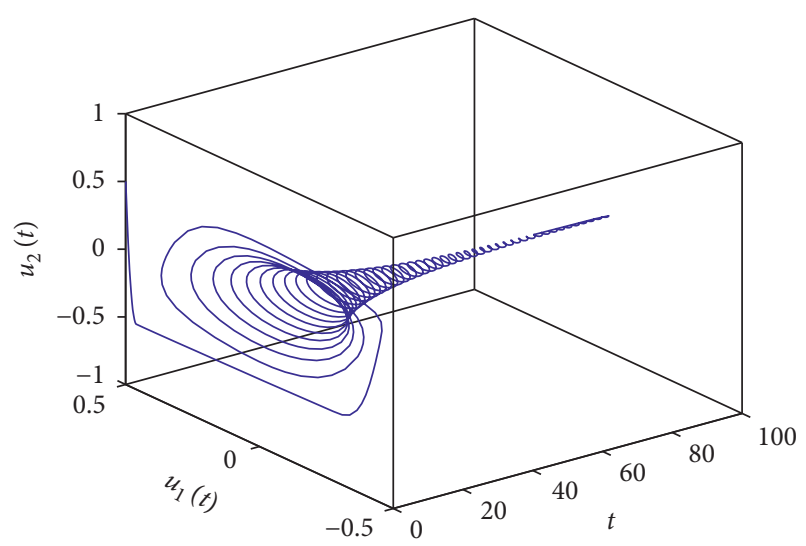

(d)

Figure 1: Simulation plots of model (62) with $\vartheta=0.12<\vartheta_{0}=0.1420$.

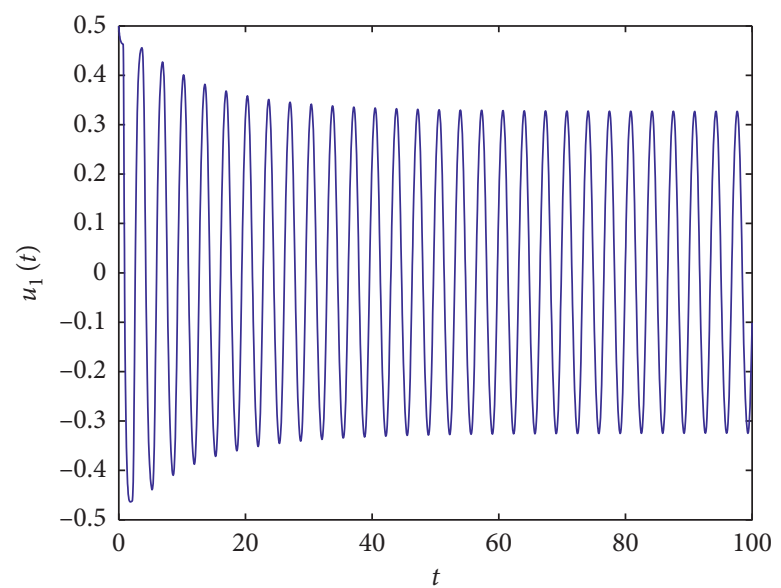

(a)

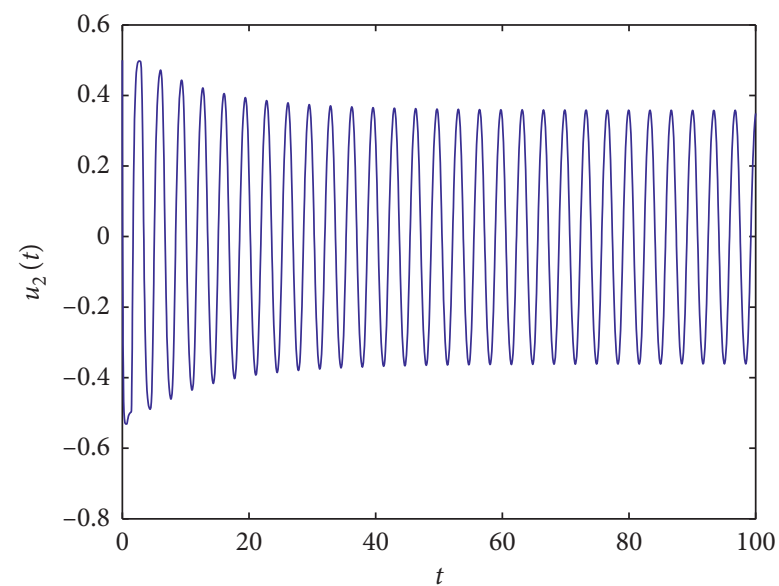

(b)

Figure 2: Continued. 


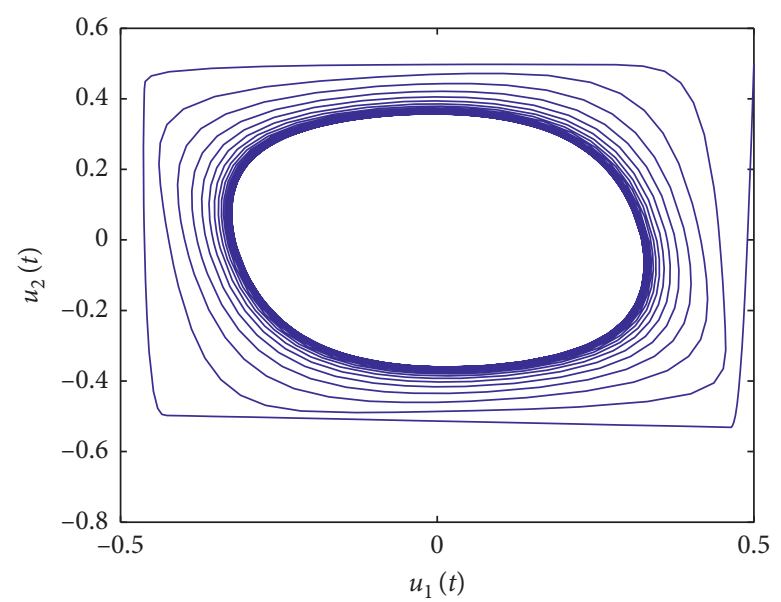

(c)

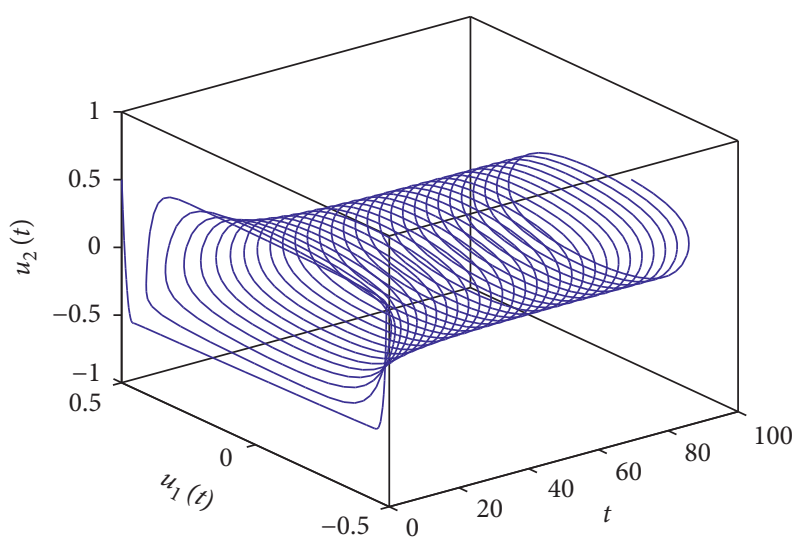

(d)

FIGURE 2: Simulation plots of model (62) with $\vartheta=0.23>\vartheta_{0}=0.1420$.

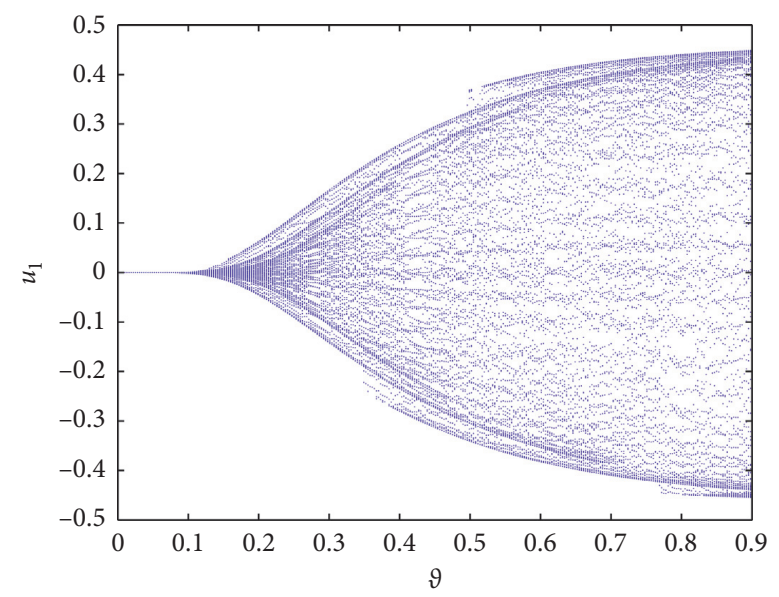

FIgURE 3: Bifurcation plot of model (62): $\vartheta-u_{1}$.

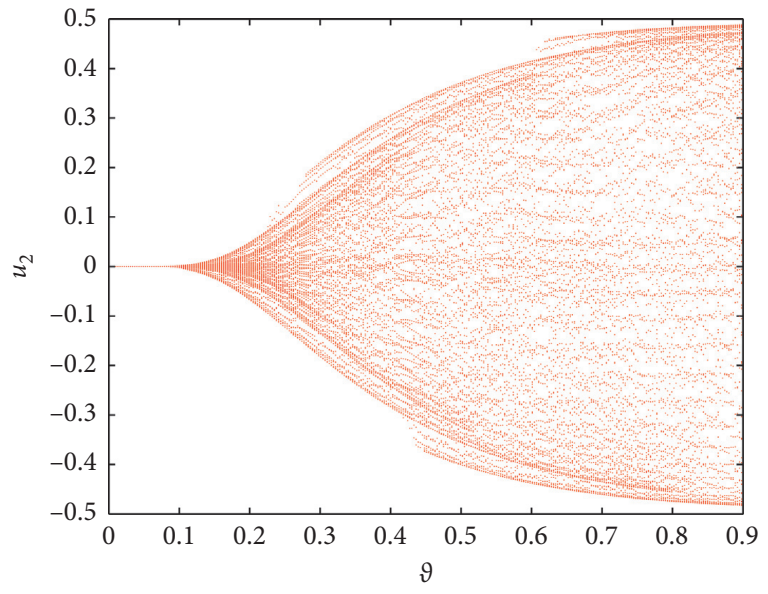

Figure 4: Bifurcation plot of model (62): $\vartheta-u_{2}$.
TABle 1: The change plot of $\xi_{0}$ and $\vartheta_{0}$ for model (62).

\begin{tabular}{lc}
\hline$\xi_{0}$ & $\vartheta_{0}$ \\
\hline 0.0088 & 0.0251 \\
0.0395 & 0.1124 \\
0.0499 & 0.1420 \\
0.1214 & 0.3455 \\
0.1471 & 0.4188 \\
0.1764 & 0.5022 \\
0.2506 & 0.7135 \\
\hline
\end{tabular}

Clearly, system (63) possesses the equilibrium point $\mathscr{E}(0,0)$. Using the computer software, one derives $\varrho_{0}=0.0955$ and $\vartheta_{1}=0.2380$ and the hypotheses (Q1) - (Q3) and (Q5) in Theorem 2 are fulfilled. So, one knows that $\mathscr{E}(0,0)$ of model (63) maintains locally 


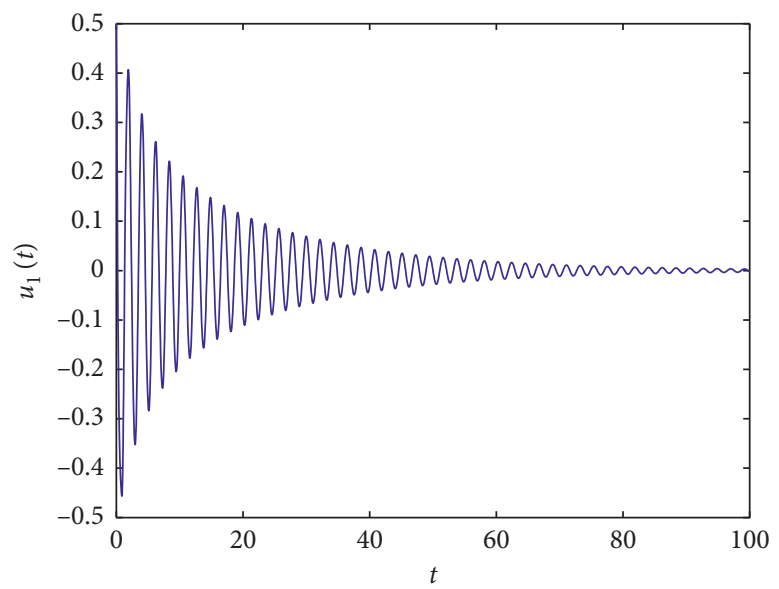

(a)

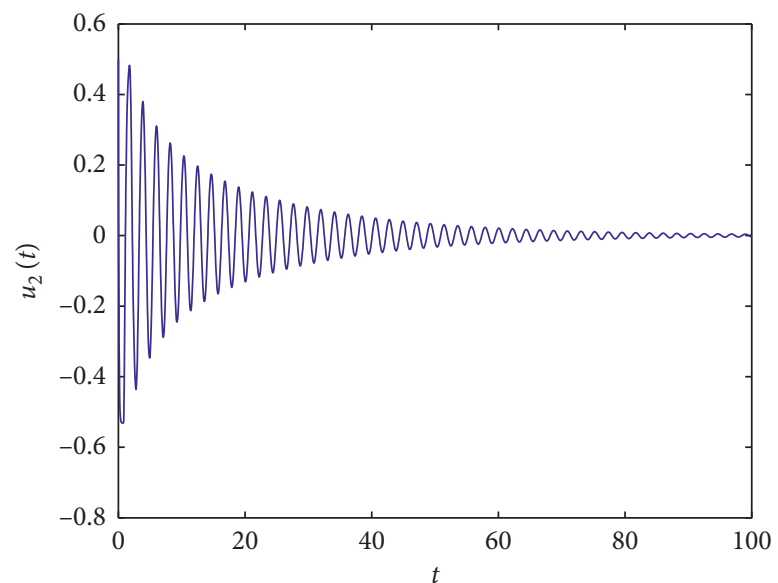

(b)

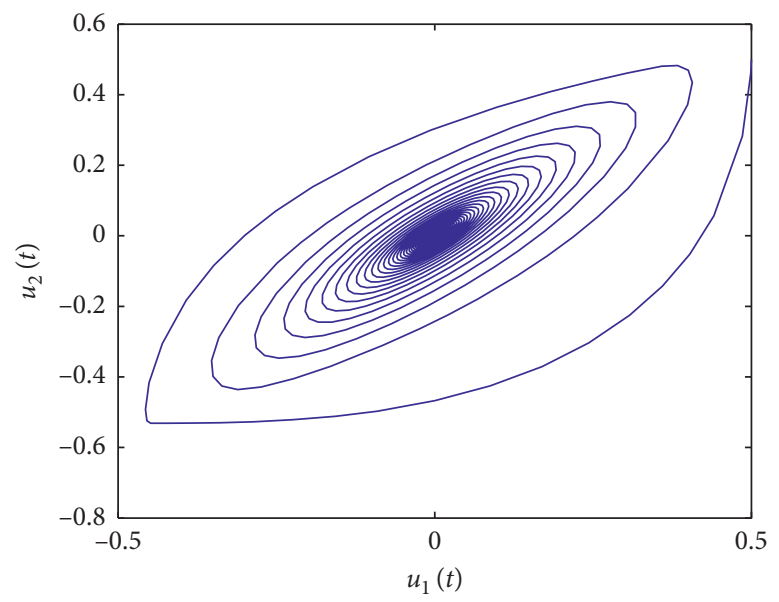

(c)

Figure 5: Continued. 


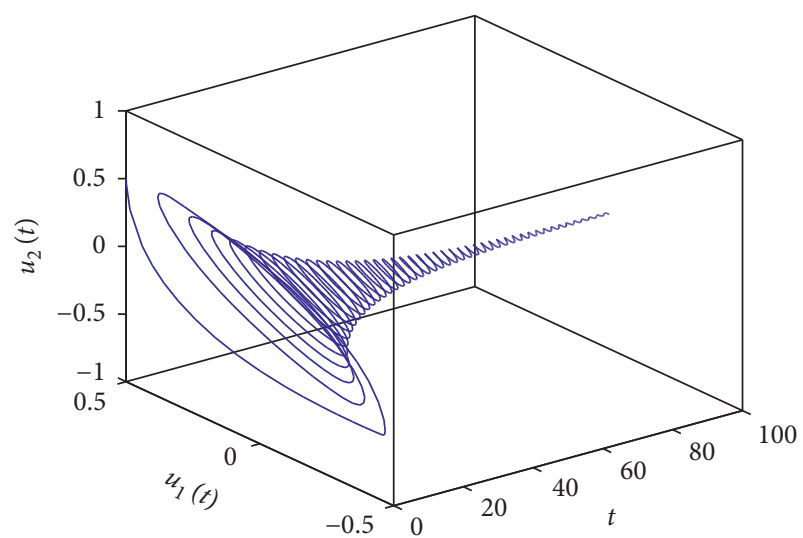

(d)

FIGURE 5: Simulation plots of model (63) with $\vartheta_{1}=0.16<\vartheta_{10}=0.2380$.

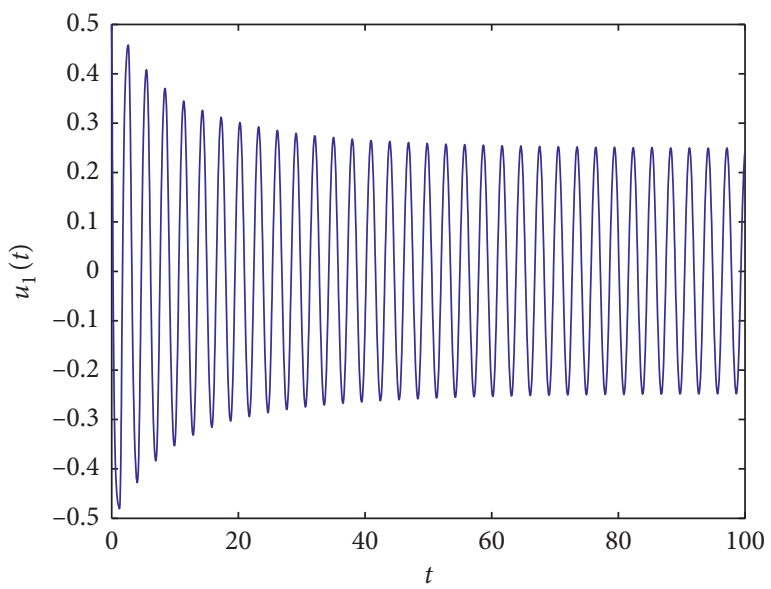

(a)

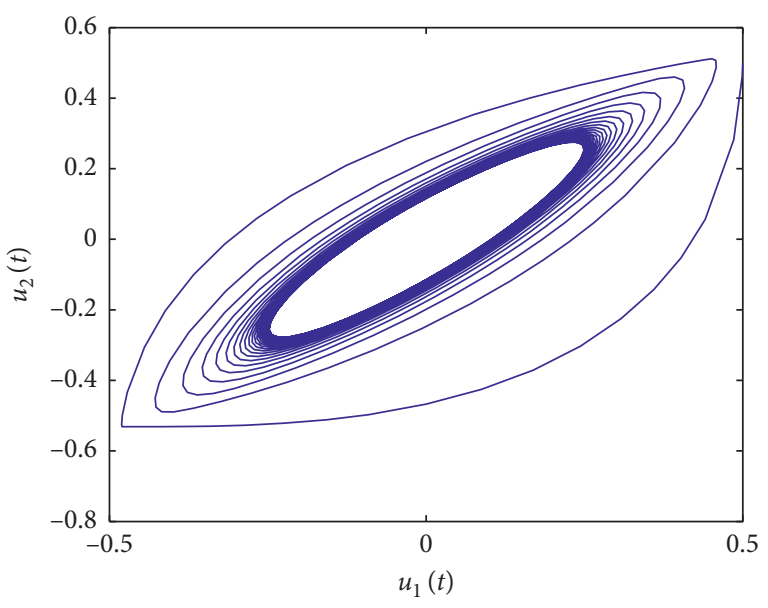

(c)

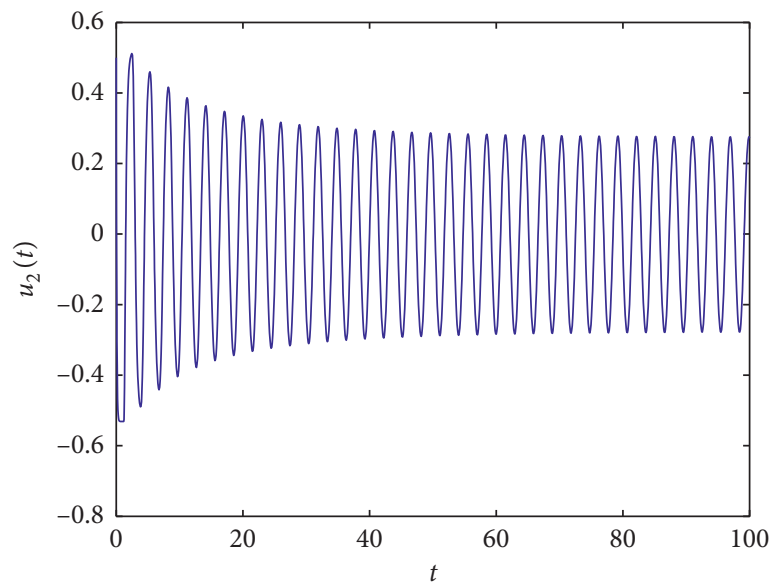

(b)

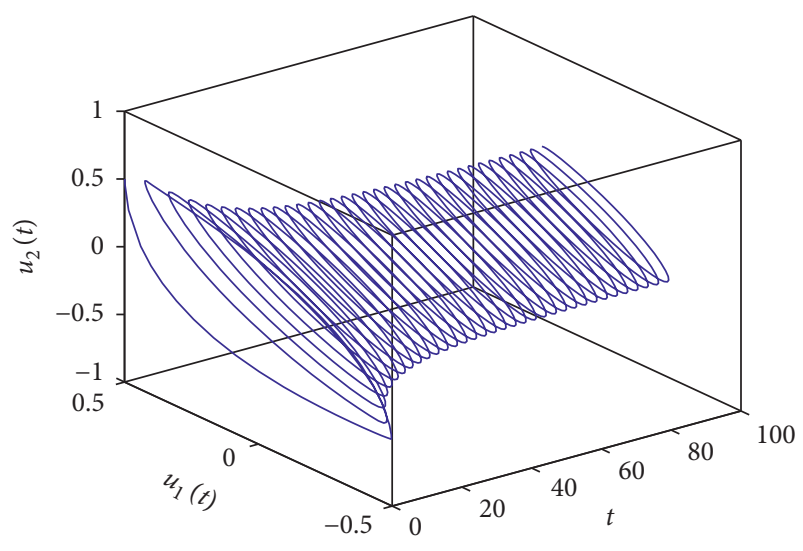

(d)

FIgURe 6: Simulation plots of model (63) with $\vartheta_{1}=0.28>\vartheta_{10}=0.2380$. 


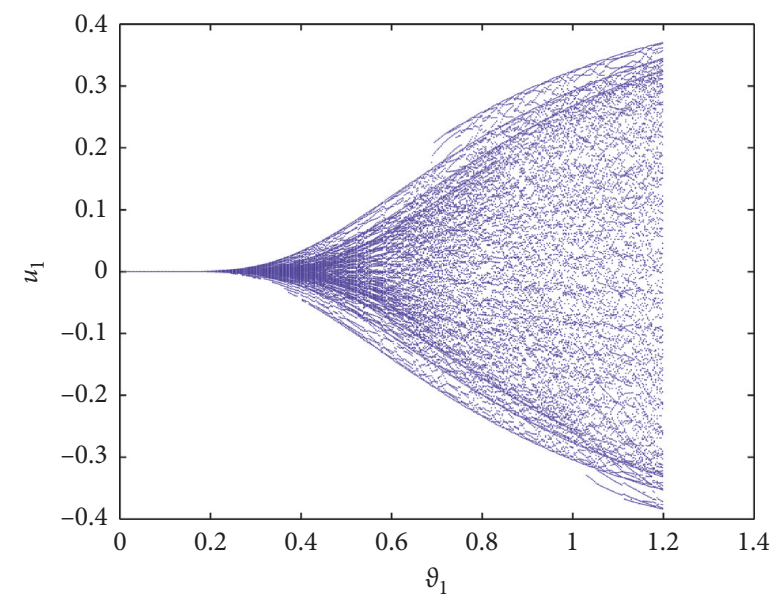

FIgURE 7: Bifurcation plot of model (63): $\vartheta_{1}-u_{1}$.

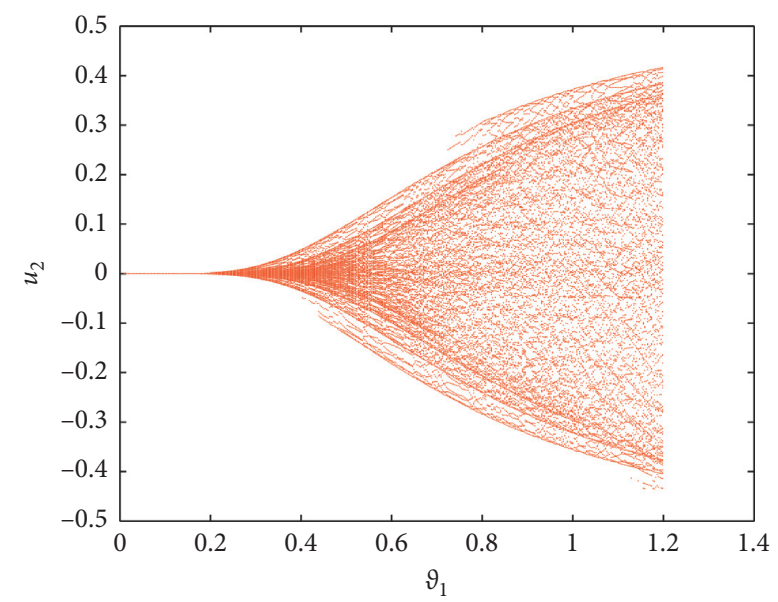

FIgURE 8: Bifurcation plot of model (63): $\vartheta_{1}-u_{2}$.

TABle 2: The change plot of $\varrho_{0}$ and $\vartheta_{10}$ of model (63).

\begin{tabular}{lc}
\hline$\varrho_{0}$ & $\vartheta_{10}$ \\
\hline 0.0362 & 0.0902 \\
0.0526 & 0.1311 \\
0.0955 & 0.2380 \\
0.1243 & 0.3098 \\
0.2218 & 0.5531 \\
0.2456 & 0.6123 \\
0.2847 & 0.7099 \\
\hline
\end{tabular}

asymptotically stable state provided that $0 \leq \vartheta_{1}<0.2380$. Choosing $\vartheta_{1}=0.16<\vartheta_{10}=0.2380$, we get the simulation plot (Figure 5) which manifests that the states of two neurons will tend to be zero. Model (63) will be unstable, and the Hopf bifurcation phenomenon takes place provided that $\vartheta_{1} \geq 0.142$. Selecting $\vartheta_{1}=0.28>\vartheta_{10}=0.2380$, one gets the simulation plot (Figure 6) which shows that the states of two neurons will keep periodic vibration in the vicinity of $\mathscr{E}(0,0)$. We give bifurcation plots (see Figures 7 and 8 ) to display the bifurcation point. Besides, the quantity change for $\varrho_{0}$ and $\vartheta_{0}$ is shown in Table 2 .

Example 3. Given the following system: $\left\{\begin{array}{l}\frac{\mathrm{d}^{\psi} u_{1}(t)}{\mathrm{d} t^{\psi}}=-\left[4.25+\sin u_{1}(t)\right]\left[1.22 u_{1}(t)-1.23 \tanh \left(u_{2}\left(t-\vartheta_{2}\right)\right)\right], \\ \frac{\mathrm{d}^{\psi} u_{2}(t)}{\mathrm{d} t^{\psi}}=-\left[6.13+\sin u_{2}(t)\right]\left[2.13 u_{2}(t)-2.05 \tanh \left(u_{1}(t)\right)\right] .\end{array}\right.$ 


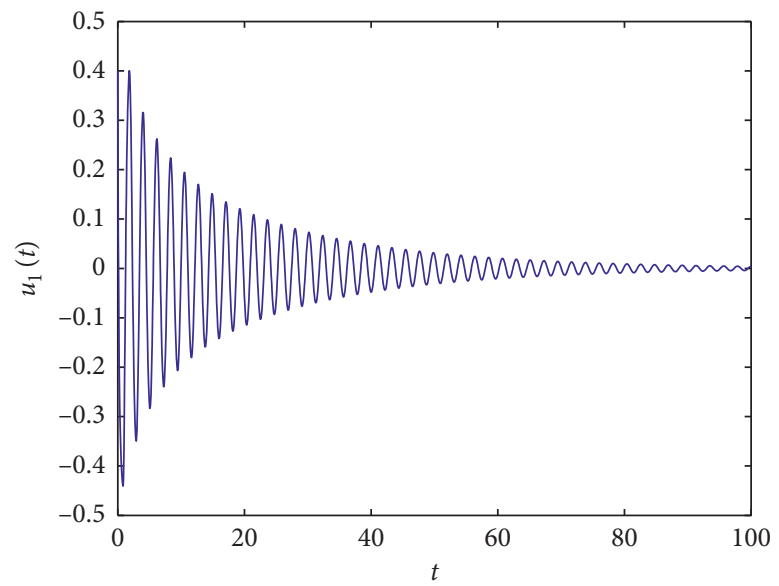

(a)

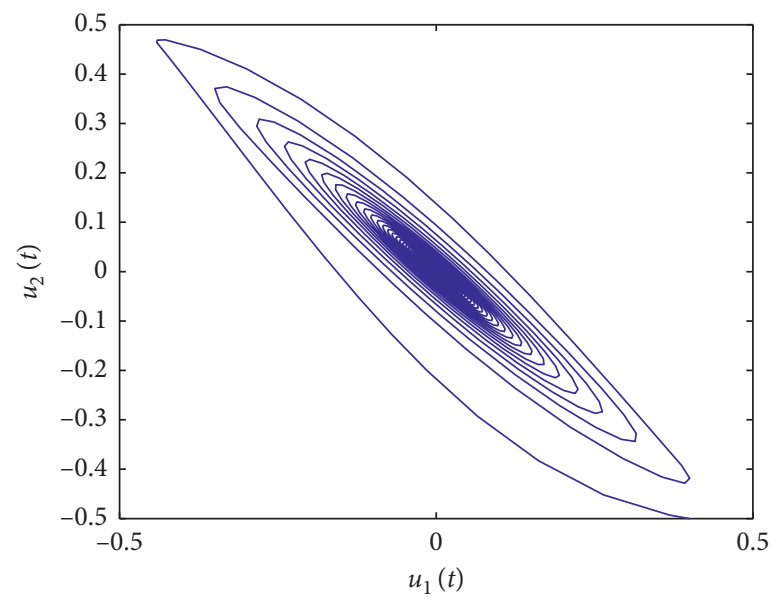

(c)

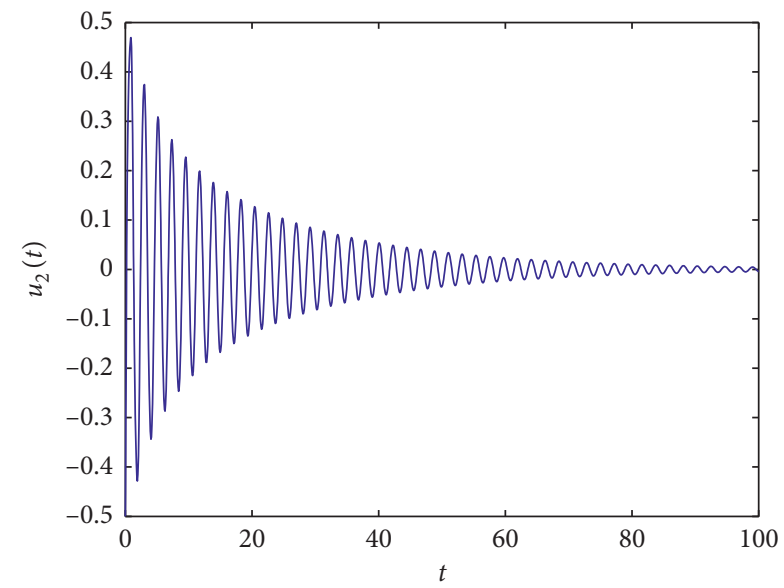

(b)

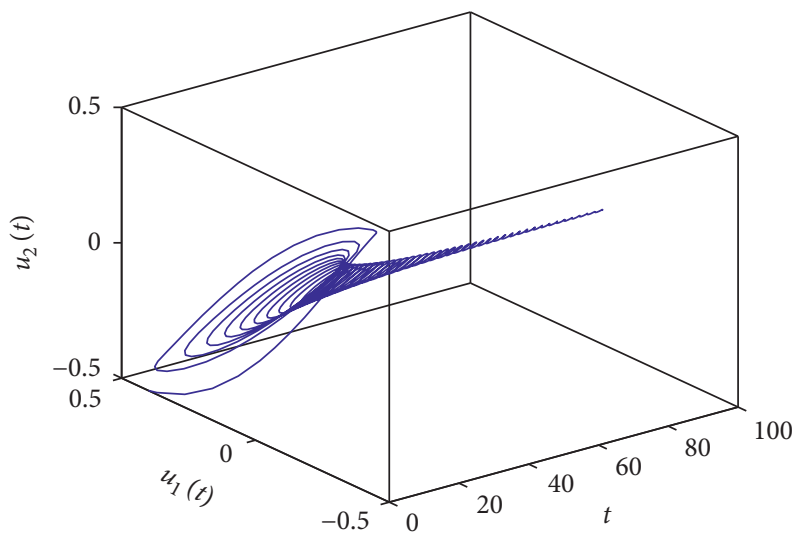

(d)

FIGURE 9: Simulation plots of model (64) with $\vartheta_{2}=0.18<\vartheta_{20}=0.2380$.

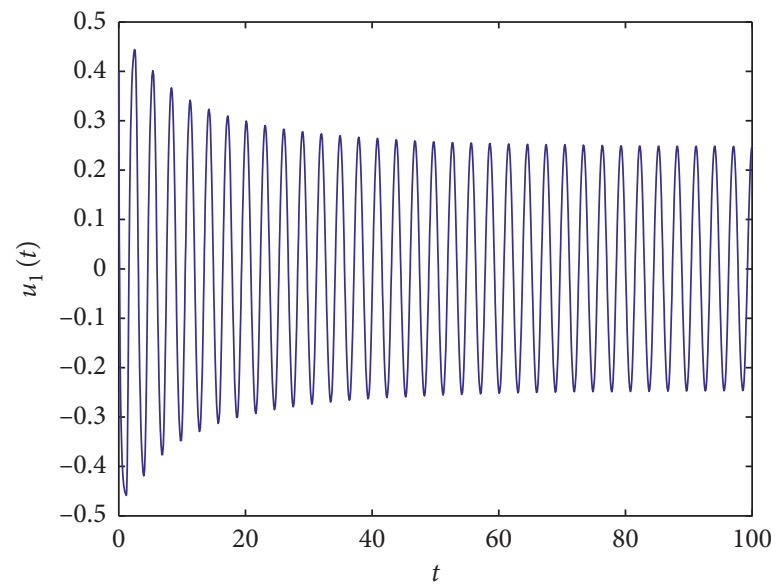

(a)

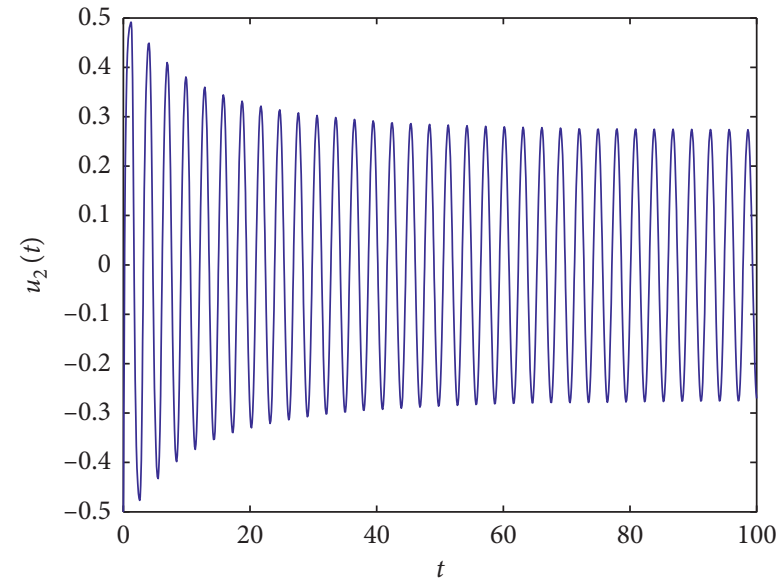

(b)

Figure 10: Continued. 


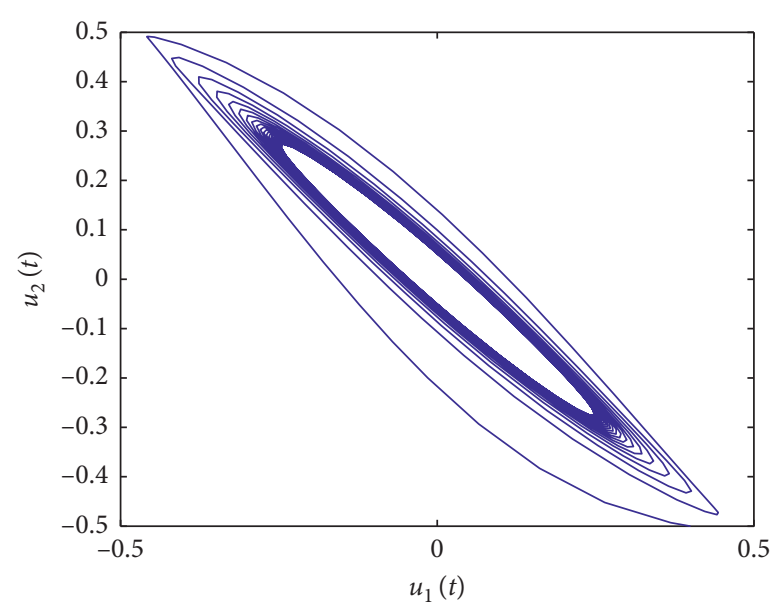

(c)

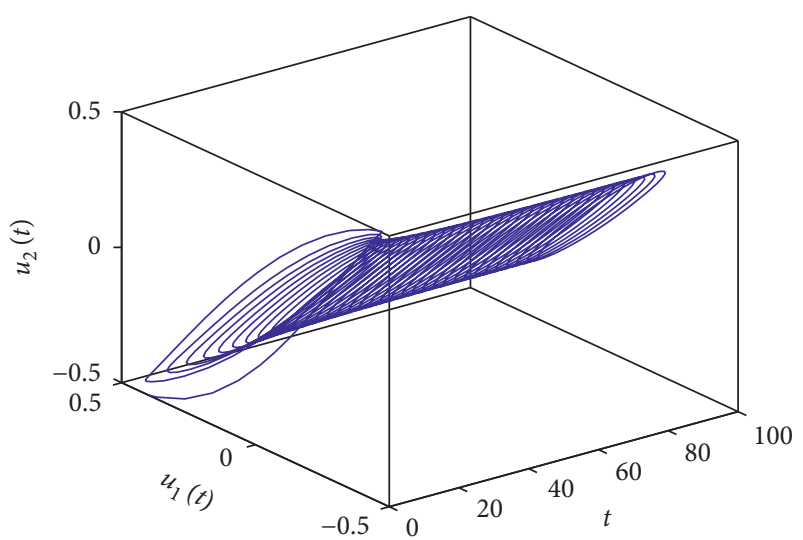

(d)

FIGURE 10: Simulation plots of model (64) with $\vartheta_{2}=0.31>\vartheta_{20}=0.2380$.

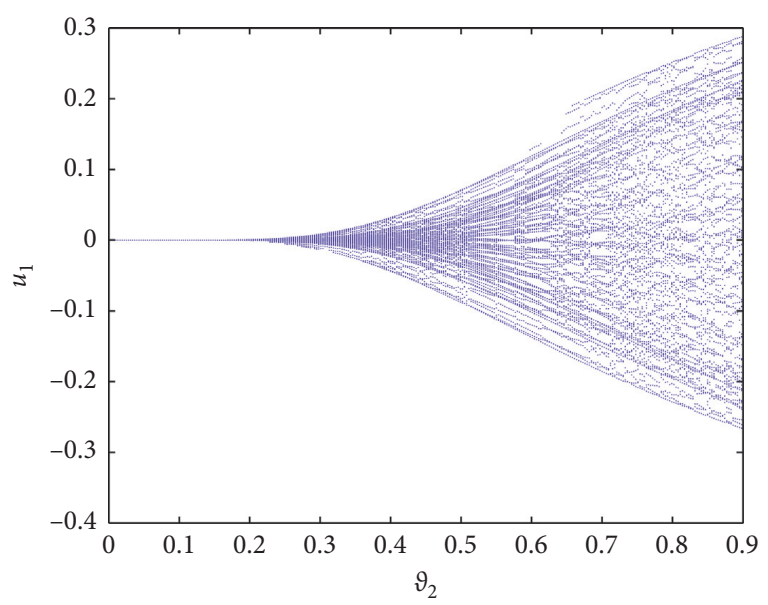

Figure 11: Bifurcation plot of model (64): $\vartheta_{2}$ versus $u_{1}$.

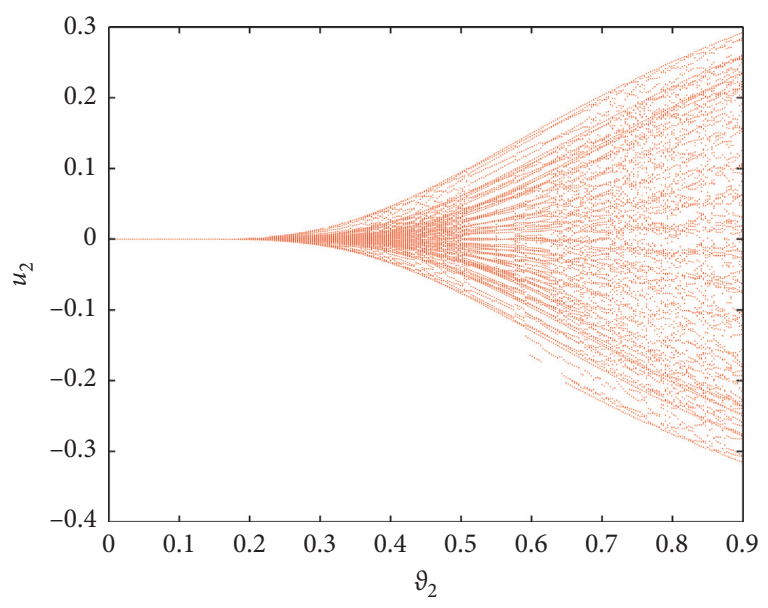

FIGURE 12: Bifurcation plot of model (64): $\vartheta_{2}$ versus $u_{2}$.
TABle 3: The change plot of $\sigma_{0}$ and $\vartheta_{20}$ of model (64).

\begin{tabular}{lc}
\hline$\sigma_{0}$ & $\vartheta_{20}$ \\
\hline 0.0322 & 0.0788 \\
0.0423 & 0.1034 \\
0.0973 & 0.2380 \\
0.1253 & 0.3065 \\
0.2585 & 0.6322 \\
0.2884 & 0.7055 \\
0.3358 & 0.8213 \\
\hline
\end{tabular}

Clearly, system (64) possesses the equilibrium point $\mathscr{E}(0,0)$. Using the computer software, one derives $\sigma_{0}=0.0973$ and $\vartheta_{20}=0.2380$ and the hypotheses $(\mathbb{Q} 1)-$ (Q3) and (Q6) in Theorem 3 are fulfilled. So, one knows that $\mathscr{E}(0,0)$ of model $(64)$ maintains locally asymptotically stable state provided that $0 \leq \vartheta_{2}<0.2380$. Choosing $\vartheta_{2}=0.18<\vartheta_{20}=0.2380$, we get the simulation plot (Figure 9) which manifests that the states of two neurons will tend to be zero. Model (63) will be unstable, and the Hopf bifurcation phenomenon takes place provided that $\vartheta_{2} \geq 0.2380$. Selecting $\vartheta_{2}=0.31>\vartheta_{20}=0.2380$, one gets the simulation plot (Figure 10) which shows that the states of two neurons will keep periodic vibration in the vicinity of $\mathscr{E}(0,0)$. We give bifurcation plots (see Figures 11 and 12 ) to display the bifurcation point. Besides, the quantity change for $\sigma_{0}$ and $\vartheta_{20}$ is shown in Table 3 .

\section{Conclusions}

As everyone knows, the stability nature and Hopf bifurcation of fractional-order models have become a focus problem in dynamical systems. In this study, we handle the stability and the emergence of Hopf bifurcation of a class of fractional-order delayed Cohen-Grossberg neural networks under three delay cases: (1) $\vartheta_{1}=\vartheta_{2}=9$; (2) $\vartheta_{2}=0$; 
and (3) $\vartheta_{1}=0$. The investigation shows that the delay plays a crucial role in stabilizing and generating Hopf bifurcation for the addressed network model. Three differential sufficient criteria are set up to guarantee the stability and the emergence of Hopf bifurcation of the considered neural networks under different cases. The software simulations verify the established theoretical findings. The derived conclusions replenish and consummate the earlier publications. The derived results can also help us manage the operation of the neural networks. In the near future, we will study the Hopf bifurcation with multiple delays and consider the Hopf bifurcation by choosing other parameters as bifurcation parameters. Also, we will deal with the Hopf bifurcation of fractional-order neural networks with different orders.

\section{Data Availability}

No data were used to support this study.

\section{Conflicts of Interest}

The author declares that there are no conflicts of interest.

\section{Acknowledgments}

This study was supported by the Social Science Fund Project of Jiangsu Institute of Technology (no. KYY17504).

\section{References}

[1] R. Curtu, "Singular Hopf bifurcations and mixed-mode oscillations in a two-cell inhibitory neural network," Physica D: Nonlinear Phenomena, vol. 239, no. 91, pp. 504-514, 2010.

[2] W. W. Yu and J. D. Cao, "Stability and Hopf bifurcation analysis on a four-neuron BAM neural network with time delays," Physics Letters A, vol. 351, no. 1-2, pp. 64-78, 2006.

[3] Y. L. Song, M. A. Han, and J. J. Wei, "Stability and Hopf bifurcation analysis on a simplified BAM neural network with delays," Physica D: Nonlinear Phenomena, vol. 200, no. 3-4, pp. 185-204, 2005.

[4] C. Xu, X. Tang, and M. Liao, "Frequency domain analysis for bifurcation in a simplified tri-neuron BAM network model with two delays," Neural Networks, vol. 23, no. 7, pp. 872-880, 2010.

[5] C. Xu, Q. Zhang, and Y. Wu, "Existence and stability of pseudo almost periodic solutions for shunting inhibitory cellular neural networks with neutral type delays and timevarying leakage delays," Network: Computation in Neural Systems, vol. 25, no. 4, pp. 168-192, 2014.

[6] C. Xu and Q. Zhang, "On antiperiodic solutions for cohengrossberg shunting inhibitory neural networks with timevarying delays and impulses," Neural Computation, vol. 26, no. 10, pp. 2328-2349, 2014.

[7] A. Hajihosseini, G. R. Rokni Lamooki, B. Beheshti, and F. Maleki, "The Hopf bifurcation analysis on a time-delayed recurrent neural network in the frequency domain," Neurocomputing, vol. 73, no. 4-6, pp. 991-1005, 2010.

[8] J. Z. Lin, R. Xu, and L. C. Li, "Turing-Hopf bifurcation of reaction-diffusion neural networks with leakage delay," Communications in Nonlinear Science and Numerical Simulation, vol. 85, Article ID 105241, 2020.
[9] C. Aouiti, E. A. Assali, I. B. Gharbia, and Y. E. Foutayeni, "Existence and exponential stability of piecewise pseudo almost periodic solution of neutral-type inertial neural networks with mixed delay and impulsive perturbations," Neurocomputing, vol. 357, pp. 292-309, 2019.

[10] C. J. Xu, M. X. Liao, P. L. Li, Z. X. Liu, and S. Yuan, "New results on pseudo almost periodic solutions of quaternionvalued fuzzy cellular neural networks with delays," Fuzzy Sets and Systems, 2020, In press.

[11] R. Kumar and S. Das, "Exponential stability of inertial BAM neural network with time-varying impulses and mixed timevarying delays via matrix measure approach," Communications in Nonlinear Science and Numerical Simulation, vol. 81, Article ID 105016, 2020.

[12] D. H. Li, Z. Q. Zhang, and X. L. Zhang, "Periodic solutions of discrete-time Quaternion-valued BAM neural networks Chaos," Solitons \& Fractals, vol. 138, Article ID 110144, 2020.

[13] M. Yan and M. Jiang, "Synchronization with general decay rate for memristor-based BAM neural networks with distributed delays and discontinuous activation functions," Neurocomputing, vol. 387, pp. 221-240, 2020.

[14] A. Abdurahman and H. Jiang, "Nonlinear control scheme for general decay projective synchronization of delayed memristor-based BAM neural networks," Neurocomputing, vol. 357, pp. 282-291, 2019.

[15] M. S. Ali, J. Yogambigai, S. Saravanan, and S. Elakkia, "Stochastic stability of neutral-type Markovian-jumping BAM neural networks with time varying delays," Journal of Computational and Applied Mathematics, vol. 349, pp. 142-156, 2019.

[16] Q. Song, L. Long, Z. Zhao, Y. Liu, and F. E. Alsaadi, "Stability criteria of quaternion-valued neutral-type delayed neural networks," Neurocomputing, vol. 412, pp. 287-294, 2020.

[17] F. Kong, Q. Zhu, and R. Sakthivel, "Finite-time and fixed-time synchronization control of fuzzy Cohen-Grossberg neural networks," Fuzzy Sets and Systems, vol. 394, pp. 87-109, 2020.

[18] Z. Ye, C. Hu, L. He, G. Ouyang, and F. Wen, "The dynamic time-frequency relationship between international oil prices and investor sentiment in China: a wavelet coherence analysis," The Energy Journal, vol. 41, no. 1, 2020.

[19] C. Huang and Y. Tan, "Global behavior of a reaction-diffusion model with time delay and Dirichlet condition," Journal of Differential Equations, vol. 271, pp. 186-215, 2021.

[20] A. Pratap, R. Raja, J. Alzabut, J. Cao, G. Rajchakit, and C. Huang, "Mittag-Leffler stability and adaptive impulsive synchronization of fractional order neural networks in quaternion field," Mathematical Methods in the Applied Sciences, vol. 43, no. 10, pp. 6223-6253, 2020.

[21] C. Song, S. Fei, J. Cao, and C. Huang, "Robust synchronization of fractional-order uncertain chaotic systems based on output feedback sliding mode control," Mathematics, vol. 7, no. 7, p. 599, 2019.

[22] C. Huang, H. Zhang, J. Cao, and H. Hu, "Stability and Hopf bifurcation of a delayed prey-predator model with disease in the predator," International Journal of Bifurcation and Chaos, vol. 29, no. 7, Article ID 1950091, 2019.

[23] C. X. Huang, H. D. Yang, and J. D. Cao, "Weighted pseudo almost periodicity of multi-proportional delayed shunting inhibitory cellular neural networks with D operator," Discrete and Continuous Dynamical Systems Series S, 2020.

[24] A. Pratap, R. Raja, C. Sowmiya, O. Bagdasar, J. Cao, and G. Rajchakit, "Robust generalized Mittag-Leffler synchronization of fractional order neural networks with discontinuous activation and impulses," Neural Networks, vol. 103, pp. 128-141, 2018. 
[25] A. Wu, L. Liu, T. Huang, and Z. Zeng, "Mittag-Leffler stability of fractional-order neural networks in the presence of generalized piecewise constant arguments," Neural Networks, vol. 85, pp. 118-127, 2017.

[26] I. Stamova and G. Stamov, "Mittag-Leffler synchronization of fractional neural networks with time-varying delays and reaction-diffusion terms using impulsive and linear controllers," Neural Networks, vol. 96, pp. 22-32, 2017.

[27] C. D. Huang, H. Liu, X. Y. Shi et al., "Bifurcations in a fractional-order neural network with multiple leakage delays," Neural Networks, vol. 131, pp. 115-126, 2020, In press.

[28] C. D. Huang, H. Liu, X. P. Chen, J. D. Cao, and A. Alsaedi, "Extended feedback and simulation strategies for a delayed fractional-order control system," Physica A: Statistical Mechanics and Its Applications, vol. 545, Article ID 123127, 2020.

[29] C. Xu, M. Liao, P. Li, Y. Guo, Q. Xiao, and S. Yuan, "Influence of multiple time delays on bifurcation of fractional-order neural networks," Applied Mathematics and Computation, vol. 361, pp. 565-582, 2019.

[30] P. Liu, M. Kong, M. Xu, J. Sun, and N. Liu, "Pinning synchronization of coupled fractional-order time-varying delayed neural networks with arbitrary fixed topology," Neurocomputing, vol. 400, pp. 46-52, 2020.

[31] X. You, Q. Song, and Z. Zhao, "Existence and finite-time stability of discrete fractional-order complex-valued neural networks with time delays," Neural Networks, vol. 123, pp. 248-260, 2020.

[32] S. Eshaghi, R. Khoshsiar Ghaziani, and A. Ansari, "Hopf bifurcation, chaos control and synchronization of a chaotic fractional-order system with chaos entanglement function," Mathematics and Computers in Simulation, vol. 172, pp. 321-340, 2020.

[33] J. Yuan, L. Zhao, C. Huang, and M. Xiao, "Novel results on bifurcation for a fractional-order complex-valued neural network with leakage delay," Physica A: Statistical Mechanics and Its Applications, vol. 514, pp. 868-883, 2019.

[34] M. Xiao, W. X. Zheng, J. Lin, G. Jiang, L. Zhao, and J. Cao, "Fractional-order PD control at Hopf bifurcations in delayed fractional-order small-world networks," Journal of the Franklin Institute, vol. 354, no. 17, pp. 7643-7667, 2017.

[35] C. Huang, J. Cao, M. Xiao, A. Alsaedi, and F. E. Alsaadi, "Controlling bifurcation in a delayed fractional predator-prey system with incommensurate orders," Applied Mathematics and Computation, vol. 293, pp. 293-310, 2017.

[36] C. Huang, T. Li, L. Cai, and J. Cao, "Novel design for bifurcation control in a delayed fractional dual congestion model," Physics Letters A, vol. 383, no. 5, pp. 440-445, 2019.

[37] C. Huang, Z. Li, D. Ding, and J. Cao, "Bifurcation analysis in a delayed fractional neural network involving self-connection," Neurocomputing, vol. 314, pp. 186-197, 2018.

[38] J. Alidousti, "Stability and bifurcation analysis for a fractional prey-predator scavenger model," Applied Mathematical Modelling, vol. 81, pp. 342-355, 2020.

[39] Q. Liu and R. Xu, "Stability and bifurcation of a CohenGrossberg neural network with discrete delays," Applied Mathematics and Computation, vol. 218, no. 6, pp. 2850-2862, 2011.

[40] I. Podlubny, Fractional Differential Equations, Academic Press, New York, NY, USA, 1999.

[41] B. Bandyopadhyay and S. Kamal, Stabliization and Control of Fractional Order Systems: A Sliding Mode Approach, Springer, Heidelberg, Germany, 2015.

[42] D. Matignon, "Stability results for fractional differential equations with applications to control processing,"
Computational Engineering in Systems Applications, vol. 2, pp. 963-968, 1996.

[43] X. Wang, Z. Wang, and J. Xia, "Stability and bifurcation control of a delayed fractional-order eco-epidemiological model with incommensurate orders," Journal of the Franklin Institute, vol. 356, no. 15, pp. 8278-8295, 2019.

[44] W. Deng, C. Li, and J. Lü, "Stability analysis of linear fractional differential system with multiple time delays," Nonlinear Dynamics, vol. 48, no. 4, pp. 409-416, 2007.

[45] C. Xu, P. Li, M. Liao, and S. Yuan, "Bifurcation analysis for a fractional-order chemotherapy model with two different delays," Mathematical Methods in the Applied Sciences, vol. 43, no. 3, pp. 1053-1083, 2020. 